\title{
Prediction of rainfall intensity measurement errors using commercial microwave communication links
}

\author{
A. Zinevich ${ }^{1}$, H. Messer ${ }^{2}$, and P. Alpert ${ }^{3}$ \\ ${ }^{1}$ The Porter School of Environmental Studies, Tel Aviv University, Israel \\ ${ }^{2}$ School of Electrical Engineering, Tel Aviv University, Israel \\ ${ }^{3}$ Department of Geophysics and Planetary Sciences, Tel Aviv University, Israel
}

Received: 1 March 2010 - Published in Atmos. Meas. Tech. Discuss.: 4 June 2010

Revised: 5 September 2010 - Accepted: 6 September 2010 - Published: 12 October 2010

\begin{abstract}
Commercial microwave radio links forming cellular communication networks are known to be a valuable instrument for measuring near-surface rainfall. However, operational communication links are more uncertain relatively to the dedicated installations since their geometry and frequencies are optimized for high communication performance rather than observing rainfall. Quantification of the uncertainties for measurements that are non-optimal in the first place is essential to assure usability of the data.

In this work we address modeling of instrumental impairments, i.e. signal variability due to antenna wetting, baseline attenuation uncertainty and digital quantization, as well as environmental ones, i.e. variability of drop size distribution along a link affecting accuracy of path-averaged rainfall measurement and spatial variability of rainfall in the link's neighborhood affecting the accuracy of rainfall estimation out of the link path. Expressions for root mean squared error (RMSE) for estimates of path-averaged and point rainfall have been derived. To verify the RMSE expressions quantitatively, path-averaged measurements from 21 operational communication links in 12 different locations have been compared to records of five nearby rain gauges over three rainstorm events.

The experiments show that the prediction accuracy is above $90 \%$ for temporal accumulation less than $30 \mathrm{~min}$ and lowers for longer accumulation intervals. Spatial variability in the vicinity of the link, baseline attenuation uncertainty and, possibly, suboptimality of wet antenna attenuation model are the major sources of link-gauge discrepancies. In addition, the dependence of the optimal coefficients of a conventional wet antenna attenuation model on spatial rainfall variability and, accordingly, link length has been shown.
\end{abstract}

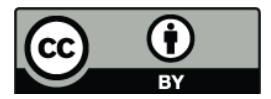

Correspondence to: A. Zinevich (artemsin@post.tau.ac.il)
The expressions for RMSE of the path-averaged rainfall estimates can be useful for integration of measurements from multiple heterogeneous links into data assimilation algorithms.

\section{Introduction}

Electromagnetic waves, especially at high (tens of GHz) radio frequencies are known to be affected by atmospheric conditions in general and by precipitation in particular. The specific rainfall-induced attenuation $K\left[\mathrm{~dB} \mathrm{~km}^{-1}\right]$ of a radio signal at the frequencies of tens of $\mathrm{GHz}$ is dominated by the effects of rainfall $R\left[\mathrm{~mm} \mathrm{~h}^{-1}\right]$ and is governed by a well-known power law equation

$K=a R^{b}$

where the parameters $a$ and $b$ are, in general, functions of link frequency, polarization and drop size distribution (DSD) (Jameson, 1991). Rainfall estimation using microwave links has been studied over the last few decades (for example, Atlas and Ulbrich 1977; an overview can be found in Zinevich et al., 2009), but only recently (Messer et al., 2006; Leijnse et al., 2007a) it has been demonstrated that data recorded in commercial cellular communication networks can be used to estimate space-time rainfall intensities.

Microwave links, being an indirect rainfall measurement tool, suffer from inherent inaccuracies. It was shown (Atlas and Ulbrich, 1977) that at the frequencies of about $35 \mathrm{GHz}$, the power-law relationship is approximately linear and is essentially independent of DSD and temperature, showing empirical errors of less than $10 \%$. However, the uncertainties in determination of path-averaged rainfall intensity due to variation in DSD increase with lowering frequency to $9 \mathrm{GHz}$ to more than 20\%. Rincon and Lang (2002) have shown that the

Published by Copernicus Publications on behalf of the European Geosciences Union. 
instantaneous estimates based on power law Eq. (1) tend to overestimate the actual rainfall, especially at high rain rates where variations in DSD affect the power law measurements, even though the agreement between power law and dual frequency estimates is very good during the intervals of stratiform rain. Wet antenna attenuation has been found to have great impact on measurement accuracy (Minda and Nakamura, 2005) if this effect is not taken into account. The uncertainties in determination of clear air attenuation due to water vapor and scintillation effects also have a direct impact on measurement quality (Holt et al., 2003; Rahimi et al., 2003; David et al., 2008). The effects of raindrop canting angles, temperature, intra- and inter-storm variations of rainfall microstructure, link length and frequency, temporal sampling strategy, power resolution and wetting of antennas have been addressed by Aydin and Daisley (2002), Berne and Uijlenhoet (2007), Leijnse et al. (2007b, 2008a, b).

However, the latter studies on uncertainties have been oriented toward estimation of expected errors using a simulation framework, primarily to choose the optimal conditions for measurement of path-averaged rainfall. The simulation results represent climatological average estimates of uncertainty that do not account for inter- and intra-storm variation of rainfall intensity. The results are therefore not directly applicable for accurate on-line variance estimation that is required, for example, for assimilation of microwave rainfall measurements (Grum et al., 2005; Zinevich et al., 2009). The experimental verification of the accuracy of uncertainty quantification has received little attention by now; it has been shown by Leijnse et al. (2008a) that experimentally measured errors considerably exceed the predicted ones, since not all error sources have been taken into account.

On the other hand, commercial hardware installations are characterized by lack of control over link parameters. The links are installed in the way that maximizes communication performance rather than the accuracy of rainfall measurements; having online variance estimation is essential for accurate integration of observations from multiple links.

This work attempts to build a framework for quantitative estimation of uncertainties of path-averaged microwave rainfall measurements. The expressions for root mean squared error (RMSE) $E[e e]$ of the estimation error $e=R-\hat{R}$ for estimates $\hat{R}$ of path-averaged rainfall $R$ have been derived. The RMSE estimates take into account the major error sources: DSD variations along a link and signal variations due to antenna wetting, quantization of the signal attenuation measurements and uncertainty in the determination of the baseline (zero rainfall) attenuation. A model of rainfall spatial variation is adopted to facilitate comparison of path-averaged rainfall estimates with nearby rain gauges, still the most reliable instrument for surface rainfall measurements.

The paper is organized as follows: in Sect. 2 the model for mean squared error (MSE) of path-averaged rainfall estimation error is formulated. The calibration of model parameters is addressed in Sect. 3. The spatial rainfall variability model is described in Sect. 4. Experimental errors and predicted RMSE are studied by comparing link and gauge observations in Sect. 5. Section 6 concludes the manuscript.

\section{Uncertainty models}

A simplified model for microwave attenuation $A_{\mathrm{M}}$, measured by a radio receiver is

$A_{\mathrm{M}}=A_{0}+A_{\mathrm{R}}+A_{\mathrm{w}}+n_{q}$

where $A_{0}$ is baseline attenuation unrelated to rainfall, $A_{\mathrm{R}}$ is path-integrated rainfall-induced attenuation, $A_{\mathrm{w}}$ is excess attenuation due to wet antenna and $n_{q}$ is observation quantization noise, modeled as a uniformly distributed random variable (Widrow and Kollár, 2008) with variance

$\sigma_{q}^{2}=\frac{\Delta^{2}}{12}$

for $\Delta \mathrm{dB}$ quantization interval. Equation (3) can be assumed valid for signals $A_{\mathrm{R}}, A_{\mathrm{w}}$ with dispersion much higher than $\Delta$; note that this assumption does not hold for weak rainfall. Both $A_{\mathrm{R}}$ and $A_{\mathrm{w}}$ depend on DSD distribution along a link; besides, all components are independent.

\subsection{Uncertainties due to DSD variations}

The path-integrated rainfall-induced attenuation $A_{\mathrm{R}}$ results from absorption and scattering of electromagnetic waves by raindrops, distributed at a point $x$ along the $L \mathrm{~km}$ link as $N_{d}(D, x)$, where $D$ is the equivolumetric raindrop diameter and $Q_{\mathrm{d}}(D)$ is the extinction cross-section at given frequency and polarization (Atlas and Ulbrich, 1977):

$$
\begin{aligned}
A_{\mathrm{R}} & =0.4343 \int_{L} d x\left(\int_{D} d D N_{d}(D, x) Q_{\mathrm{d}}(D)\right) \\
& =0.4343 \int_{D} d D \bar{N}_{d}(D, L) Q_{\mathrm{d}}(D)
\end{aligned}
$$

where $\bar{N}_{d}(D, L)=\int_{L} d x N_{d}(D, x)$ is the path-integrated DSD. Similarly, the path-averaged rainfall $R_{L}$ is given by

$$
R_{L}=\frac{0.6 \pi}{L} \int_{D} d D \bar{N}_{d}(D, L) V_{\mathrm{d}}(D) D^{3},
$$

where $V_{\mathrm{d}}(D)$ is the raindrop terminal velocity. Since both $V_{\mathrm{d}}(D)$ and the scattering cross-section can be approximated by power laws $V_{\mathrm{d}}(D)=3.78 D^{0.67}, Q_{\mathrm{d}}(D)=C D^{n}$ (Atlas and Ulbrich 1977), both $A_{\mathrm{R}}$ and $R_{L}$ can be considered higher-order moments of the DSD $N_{d}(D)$. The relation between $A_{\mathrm{R}}$ and $R_{L}$ becomes linear at frequencies of about $34 \mathrm{GHz}$ where the power $n$ in the cross-section expression equals to that of $V_{\mathrm{d}}(D)$. Commercial microwave links operate at various frequencies; uncertainty in determination of 
path-averaged rain rate from attenuation measurements increases as frequency lowers (Atlas and Ulbrich, 1977).

For a given link, the stochastic relationship between $A_{\mathrm{R}}$ and $R_{L}$ can be obtained empirically by fitting their estimates based on the DSD measurements of $\bar{N}_{d}(D, L)$ for a given link length $L$. For convenience, let us write an expression for the expected value of $R_{L}$ given $A_{\mathrm{R}}$ according to Eq. (1) with inverted power law coefficients $\beta=b^{-1}, \alpha=(a L)^{-\beta}$ :

$\hat{R}_{L}\left(A_{\mathrm{R}}\right)=E\left[R_{L} \mid A_{\mathrm{R}}\right] \cong \alpha A_{\mathrm{R}}^{\beta}$

The MSE of $R_{L}$ due to DSD variations can be modeled using another ad hoc power-law expression with two linkspecific parameters $\gamma, \delta$ (we adopt a notation $\sigma^{2}[R \mid A]=$ $E\left[(R-\hat{R}(A))^{2} \mid A\right]$ for MSE):

$\hat{\sigma}_{\mathrm{DSD}}^{2}\left[R_{L} \mid A_{\mathrm{R}}\right]=E\left[\left(R_{L}-\hat{R}_{L}\left(A_{\mathrm{R}}\right)\right)^{2} \mid A_{\mathrm{R}}\right] \cong \gamma A_{\mathrm{R}}^{\delta}$.

The verification of adequacy of the power law parametric form is addressed in the context of a model, comprising wet attenuation effects, in Sect. $3, c$.

\subsection{Uncertainties due to antenna wetting}

A thin film of water on an antenna or a radome is known to cause a considerable attenuation of the received signal. A simplified empirical two-parameter model for a wet antenna attenuation estimate $\hat{A}_{\mathrm{w}}$, originating from (Kharadly and Ross, 2001) have been used by Minda and Nakamura (2005); Leijnse et al. (2007b); Zinevich et al. (2009):

$\hat{A}_{\mathrm{w}}=c_{1}\left(1-e^{-c_{2}\left(A_{\mathrm{R}}+A_{\mathrm{w}}\right)}\right)$,

Denoting $a_{\mathrm{W}}(A)=c_{1}\left(1-e^{-c_{2} A}\right)$, let us represent the true wet antenna attenuation as

$A_{\mathrm{W}}=a_{\mathrm{w}}\left(A_{\mathrm{R}}+A_{\mathrm{W}}\right)+n_{\mathrm{W}}$

where $n_{\mathrm{W}}$ is noise, caused by rainfall variations near the antennas. Substituting unknown $A_{\mathrm{R}}+A_{\mathrm{w}}$ from Eq. (2) into Eq. (8), Eq. (2) transforms into

$A_{\mathrm{R}}+n_{\mathrm{W}}=A_{\mathrm{M}}-A_{0}-n_{q}-a_{\mathrm{W}}\left(A_{\mathrm{M}}-A_{0}-n_{q}\right)$.

Since both $n_{\mathrm{w}}$ and the measurement error in $R_{L}$ are caused by DSD variability along a link, they should be modeled jointly using the same DSD data. Taking into account that the effect of $n_{\mathrm{w}}$ on rainfall estimate decreases with increase of $A_{\mathrm{R}}+n_{\mathrm{w}}$ according to Eq. (8) (for large $A_{\mathrm{M}}, A_{\mathrm{W}} \rightarrow c_{1}$ and is weakly affected by its variations), a following ad hoc parametric MSE model, parameterized by three link-specific variables $\gamma, \delta, \varepsilon$ is proposed:

$$
\begin{aligned}
\hat{\sigma}_{\mathrm{DSD}+\mathrm{Wet}}^{2}\left[R_{L} \mid A_{\mathrm{R}}+n_{\mathrm{w}}\right] & =E\left[\left(R_{L}-\hat{R}_{L}\left(A_{\mathrm{R}}+n_{\mathrm{w}}\right)\right)^{2} \mid A_{\mathrm{R}}+n_{\mathrm{w}}\right] \\
& \cong \gamma\left(A_{\mathrm{R}}+n_{\mathrm{W}}\right)^{\delta} e^{-\varepsilon\left(A_{\mathrm{R}}+n_{\mathrm{w}}\right)} .
\end{aligned}
$$

The model given by Eq. (6) can be adopted for $\hat{R}_{L}\left(A_{\mathrm{R}}+n_{\mathrm{W}}\right)$ keeping in mind that even zero-mean $n_{\mathrm{W}}$ leads to a biased estimate of $R_{L}$ since

$$
E\left[\alpha\left(A_{\mathrm{R}}+n_{\mathrm{w}}\right)^{\beta}\right] \neq E\left[\alpha A_{\mathrm{R}}^{\beta}\right]
$$

due to non-linearity of the power law Eq. (6).

\subsection{Uncertainties due to baseline variation}

The level of baseline attenuation $A_{0}(t)$, where $t$ is a time index, varies in time due to primarily variations of water vapor concentration in the atmosphere, ducting and scintillation; the transmission/reception analog circuitry is affected by temperature variations that may lead to additional signal variations (Leijnse et al., 2007b). In addition, wind effects on the antennas and masts may also cause variations in the baseline signal; the estimation of the latter is complicated by signal quantization. In this work, the baseline attenuation estimate has been calculated as a sample mean $\left(\hat{A}_{0}\right)$ of attenuation measurements immediately before and after a rainstorm: the time of rainstorm starts and ends in the area has been determined according to nearby rain gauges with $10 \mathrm{~min}$ margins, to compensate for link-gauge physical distance. Then, the measurements of $A_{\mathrm{M}}$ of $2 \ldots 27 \mathrm{~h}$ length (depending on data availability) have been used for calculations, described below. For practical applications, existing rain/no rain detection techniques can be used (Rahimi et al., 2003; Upton et al., 2005; Goldshtein et al., 2009; Schleiss and Berne, 2009). The noise $n_{0}$ due to short-time variations

$n_{0}(t)=A_{0}(t)-\hat{A}_{0}$

is zero mean under an assumption that the average baseline estimate, obtained from measurements before and after the rainstorm, gives an unbiased estimate of baseline during the rainstorm (the best guess, provided that there is no other information w.r.t. baseline variation during a rainstorm is available).

To quantify uncertainty of $\hat{A}_{0}$ (MSE of $n_{0}$ ), a sample MSE estimate $\hat{\sigma}_{0}^{2}$ has been calculated over no-rain intervals as a deviation from $\hat{A}_{0}$, assuming that baseline variations during rainy periods have similar statistical properties. Note that the length of data samples before and after event should be equal; otherwise, the sample MSE becomes biased towards the longer interval. While short data intervals may lead to inaccurate estimates of the baseline variations, they are still unbiased as long as the unbiased sample variance estimate (Papoulis, 1991) is used for sample MSE calculation. Measurement of temporally averaged rainfall requires estimation of baseline variability in the same temporal scale. To facilitate MSE prediction of accumulated rainfall amounts over an interval of $\Delta t$ samples length, the pre/post rainstorm attenuation measurements have been averaged over a sliding window of length $\Delta t$ prior to calculation of $\hat{\sigma}_{0}^{2}$ (note that averaging of rain rates is not equal to averaging attenuations, 


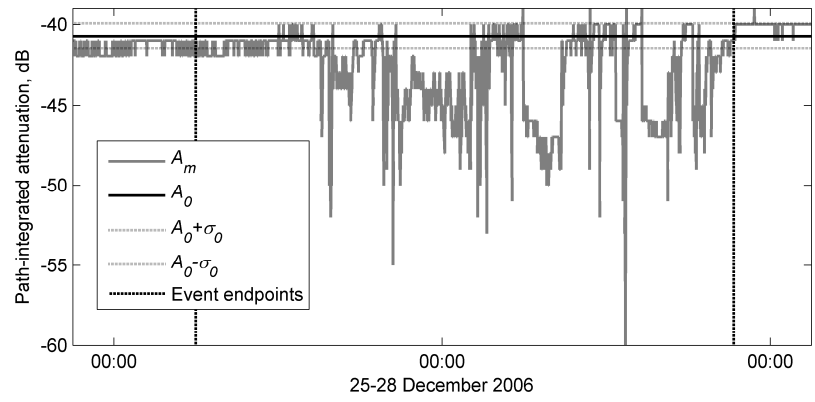

Fig. 1. An example of the baseline attenuation determination $(23.27 \mathrm{GHz}, 2.19 \mathrm{~km}) . A_{\mathrm{M}}$ is the measured attenuation, $A_{0}$ is the estimated baseline, and $\sigma_{0}$ is RMSE of $A_{0}$. Event endpoints, defining the beginning and the end of the rainstorm, are determined according to the nearby Switch Ramle gauge.

due to non-linearity of Eq. (1); these differences are of second order and are neglected for MSE prediction). An example of the baseline and MSE is given in the Fig. 1. Note that at frequencies around $34 \mathrm{GHz}$ where the attenuation-rain rate relation becomes nearly-linear, the difference between averaging of rain rate and attenuation nearly vanishes.

In some cases, the natural short-term variations in $A_{0}$ due to the atmospheric scintillation produce a dithering effect on the quantized signal so that sample mean represents the average baseline attenuation; for short $\Delta t$, quantization noise in pre/post rainstorm samples is also absorbed into $\hat{\sigma}_{0}^{2}$ that may lead to overestimation of baseline variability. For short links or at low frequencies, the natural fluctuations of the base level attenuation are comparable in magnitude to the quantization interval $\Delta=1 \mathrm{~dB}$ (in the present study). In this case, quantization causes a nonlinear distortion of the signal; the true $A_{0}$ is known to within $\Delta$. Increasing sample size does not decrease the variance of quantization error, given by Eq. (3), and the estimation of true $\hat{\sigma}_{0}^{2}$ is complicated. A possible heuristics in this case is to limit $\hat{\sigma}_{0}^{2}$ to the minimum given by Eq. (3), supposing that near-zero $\hat{\sigma}_{0}^{2}$ indicates that the nonlinear quantization effect dominates other variability sources. Because of non-linear quantization effects, quantization error in baseline estimate may affect an entire rain event, introducing a bias in estimation of path-averaged rainfall.

Substituting Eq. (13) into Eq. (10), we get the signal distortion model

$A_{\mathrm{R}}+n_{\mathrm{w}}=A_{\mathrm{M}}-\hat{A}_{0}-n_{0}-n_{q}-a_{\mathrm{w}}\left(A_{\mathrm{M}}-\hat{A}_{0}-n_{0}-n_{q}\right)$.

Note that, in general, signal quantization can be performed in different ways: for example, rounding of measured signal to a nearest integer value does not introduce bias, while flooring or ceiling do introduce negative or positive bias of $\Delta / 2 \mathrm{~dB}$. Naturally, this bias presents in both rainy signal attenuation measurement $A_{\mathrm{M}}$ and the measurements of $A_{\mathrm{M}}$, used to calculate $\hat{A}_{0}$. Because of this, the quantizationrelated bias, if any, is cancelled for $A_{\mathrm{M}}-\hat{A}_{0}$, and therefore $n_{q}$ in Eq. (14) can be considered zero-mean.

\subsection{Uncertainties of estimation of path-averaged rainfall}

Employing the Taylor series expansion of non-linear $a_{\mathrm{w}}\left(A_{\mathrm{M}}-\hat{A}_{0}-n_{0}-n_{q}\right)$ around the estimate of the rainfallinduced attenuation $A_{\mathrm{M}}-\hat{A}_{0}$ and taking linear terms, we can rewrite Eq. (14) as

$$
\begin{aligned}
& a_{\mathrm{w}}\left(A_{\mathrm{M}}-\hat{A}_{0}-n_{q}-n_{0}\right) \cong a_{\mathrm{w}}\left(A_{\mathrm{M}}-\hat{A}_{0}\right)+a_{\mathrm{w}}^{\prime} \\
&\left(A_{\mathrm{M}}-\hat{A}_{0}\right)\left(-n_{q}-n_{0}\right), \\
& A_{\mathrm{R}}+n_{\mathrm{w}} \cong A_{\mathrm{M}}-\hat{A}_{0}-a_{\mathrm{w}}\left(A_{\mathrm{M}}-\hat{A}_{0}\right)-t_{M} \cdot\left(n_{q}+n_{0}\right),
\end{aligned}
$$

where $a_{\mathrm{w}}^{\prime}$ is the first derivative of $a_{\mathrm{w}}$ w.r.t. $A_{\mathrm{M}}-\hat{A}_{0}$, and $t_{M}=\left(1-c_{1} c_{2} \exp \left(-c_{2}\left(A_{\mathrm{M}}-\hat{A}_{0}\right)\right)\right)$ is an auxiliary variable. Recalling that both $n_{q}$ and $n_{0}$ are zero-mean and independent, the estimates of the rainfall-induced attenuation $\hat{A}_{\mathrm{R}}=E\left[A_{\mathrm{R}}+n_{\mathrm{w}} \mid A_{\mathrm{M}}-\hat{A}_{0}\right]$ and its MSE become

$\hat{A}_{\mathrm{R}}=A_{\mathrm{M}}-\hat{A}_{0}-a_{\mathrm{w}}\left(A_{\mathrm{M}}-\hat{A}_{0}\right)$,

$\hat{\sigma}^{2}\left[A_{\mathrm{R}}+n_{\mathrm{w}} \mid A_{\mathrm{M}}-\hat{A}_{0}\right] \cong t_{M}^{2}\left(\frac{\Delta^{2}}{12}+\sigma_{0}^{2}\right)$.

Neglecting the higher-order terms of the Taylor series is plausible under an assumption that the magnitude of $A_{\mathrm{M}}-\hat{A}_{0}$ is much higher than that of the noise terms. While this is the case for high rain rates where $a_{\mathrm{w}}$ is nearly constant (otherwise, the reduction of Eq. (14) to the conventional Eq. (17) similar to the one used, for example, by Leijnse et al. (2007b) would be impossible), this approximation can lead to the additional errors in the estimation of $\hat{A}_{\mathrm{R}}$ and $\hat{\sigma}^{2}\left[A_{\mathrm{R}}+n_{\mathrm{W}} \mid A_{\mathrm{M}}-\hat{A}_{0}\right]$ for weak rain rates where signal $A_{\mathrm{M}}-\hat{A}_{0}$ to noise $n_{q}+n_{0}$ ratio is low and more Taylor expansion terms may be required to accurately represent $a_{\mathrm{w}}$. The second derivative $a_{\mathrm{w}}^{\prime \prime}(x)<0$; the direct consequence of this is that the conventional estimation of $\hat{A}_{\mathrm{R}}$ using Eq. (17) leads to overestimation of weak rain rates due to neglecting of the term with $a_{\mathrm{w}}^{\prime \prime}(x)$. By retaining the term with $a_{\mathrm{w}}^{\prime \prime}$, Eq. (17) transforms into

$\widehat{A}_{R} \cong A_{\mathrm{M}}-\hat{A}_{0}-a_{\mathrm{w}}\left(A_{\mathrm{M}}-\hat{A}_{0}\right)-\frac{a_{w}^{\prime \prime}\left(A_{\mathrm{M}}-\hat{A}_{0}\right)}{2} \cdot E\left[\left(n_{q}+n_{0}\right)^{2}\right]$.

One can see that zero-mean noise $n_{q}+n_{0}$ leads to a bias if the last term in Eq. (19) is dropped. However, this issue can be put aside as long as the empirical function $a_{\mathrm{w}}$ is calibrated for the model given by Eq. (17) to provide unbiased rain rate 
estimation (see Sect. 3.2). Substituting Eq. (17) into Eq. (16) and substituting $A_{\mathrm{R}}+n_{\mathrm{w}}$ for $A_{\mathrm{R}}$ in Eq. (6), we can rewrite the latter as

$\hat{R}_{L}\left(\hat{A}_{\mathrm{R}}\right)=E\left[\alpha\left(A_{\mathrm{R}}+n_{\mathrm{w}}\right)^{\beta} \mid \hat{A}_{\mathrm{R}}\right] \cong E\left[\alpha\left(\hat{A}_{\mathrm{R}}-t_{M}\left(n_{q}+n_{0}\right)\right)^{\beta}\right]$

Again, using a linear approximation around $\hat{A}_{\mathrm{R}}$, denoting another auxiliary variable $d_{M}=\alpha \beta \hat{A}_{R}^{\beta-1} t_{M}$, the Eq. (20) simplifies to a trivial

$\hat{R}_{L}\left(\hat{A}_{\mathrm{R}}\right) \cong E\left[\alpha \hat{A}_{\mathrm{R}}^{\beta}-d_{M}\left(n_{q}+n_{0}\right)\right]=\alpha \hat{A}_{\mathrm{R}}^{\beta}$.

Similarly, noticing independence of $n_{q}$ and $n_{0}$ with DSDrelated uncertainties and substituting the Eq. (11), Eq. (18) transforms into

$$
\begin{aligned}
\hat{\sigma}^{2}\left[R_{L} \mid \hat{A}_{\mathrm{R}}\right] & \cong E\left[\left(R-\alpha \hat{A}_{\mathrm{R}}^{\beta}+d_{M}\left(n_{q}+n_{0}\right)\right)^{2}\right] \cong \\
& \cong \hat{\sigma}_{\mathrm{DSD}+\mathrm{Wet}}^{2}\left(R_{L} \mid \hat{A}_{\mathrm{R}}\right)+d_{M}^{2}\left(\frac{\Delta^{2}}{12}+\hat{\sigma}_{0}^{2}\right) .
\end{aligned}
$$

\subsection{Temporal averaging}

To get a better insight into the effect of various error sources as a function of temporal averaging intervals, let us estimate the MSE of path- and time-averaged rain rate $\left\langle R_{L}(t)\right\rangle$ over $\Delta t$ minute interval, $t=1, \ldots, \Delta t$, given a set of instantaneous attenuation measurements $\Omega=\left\{A_{\mathrm{M}}(t)-\hat{A}_{0}(t) ; t=\right.$ $1, \ldots, \Delta t\}$. By substituting the averaging operator $\langle\cdot\rangle$ into Eq. (11),

$\hat{\sigma}^{2}\left[\left\langle R_{L}(t)\right\rangle \mid \Omega\right] \cong E\left[\left(\left\langle R_{L}(t)\right\rangle-\left\langle\alpha \hat{A}_{\mathrm{R}}^{(t) \beta}+d_{M}(t)\left(n_{q}(t)+n_{0}\right)\right\rangle\right)^{2}\right]$.

Here, $d_{M}(t)$ is obtained from $d_{\mathrm{M}}$ by substituting $\hat{A}_{\mathrm{R}}(t)$, $A_{\mathrm{M}}(t), \hat{A}_{0}(t)$ for $\hat{A}_{\mathrm{R}}, A_{\mathrm{M}}, \hat{A}_{0}$. Note that $n_{0}$ does not depend on $t=1, \ldots, \Delta t$ (that is, the typical period of variations of $n_{0}$ is assumed to be much longer than $\Delta t$; Eq. (23) does not account for instantaneous baseline variations due to scintillation since their effect on $\left\langle R_{L}(t)\right\rangle$ is assumed to be minor due to averaging). Rearranging terms on the r.h.s. of Eq. (23), recalling independence of $n_{q}$ and $n_{0}$ on each other and on DSD-related errors, we get

$$
\begin{aligned}
\hat{\sigma}^{2}\left[\left\langle R_{L}(t)\right\rangle \mid \Omega\right] \cong & \left\langle\hat{\sigma}_{\mathrm{DSD}+\mathrm{Wet}}^{2}\left(R_{L} \mid \hat{A}_{\mathrm{R}}(t)\right)\right\rangle+\frac{1}{\Delta t}\left\langle d_{M}(t)^{2}\right\rangle \\
& \frac{\Delta^{2}}{12}+\left\langle d_{M}(t)\right\rangle^{2} \hat{\sigma}_{0}^{2} .
\end{aligned}
$$

\subsection{Summary of the uncertainty model}

To sum up, we have derived the estimates of path-integrated rainfall-induced attenuation $\hat{A}_{\mathrm{R}}$ and path-averaged rainfall $\hat{R}_{L}$ in Eqs. (17), (21) based on the model for the measured signal $A_{\mathrm{M}}$ given by Eq. (2), comprising baseline attenuation $A_{0}$, path-integrated rainfall-induced attenuation $A_{\mathrm{R}}$, wet antenna attenuation $A_{\mathrm{w}}$ and quantization error $n_{q}$. Using the first-order approximations of nonlinear models for $A_{\mathrm{w}}$ (Eq. 8) and the power-law relation (Eq. 1), the estimate $\hat{A}_{\mathrm{R}}$ in Eq. (17) is equivalent to the deterministic relation (e.g. Leijnse et al., 2007b; Zinevich et al., 2009). Next, we have derived the Eq. (22) for MSE for path-average rainfall estimates, based on an ad hoc model for uncertainty of the $A-R$ relation given by Eq. (11), sample MSE estimate $\hat{\sigma}_{0}^{2}$ of baseline uncertainty and a simplified model for variance of quantization error (Eq. 3). Finally, Eq. (24) for MSE of time- and path-averaged rainfall has been derived.

\section{Calibration of model parameters}

The model parameters (rainfall attenuation and MSE model coefficients, wet attenuation coefficients) have been calibrated using a DSD database and a set of rain gauge and microwave links records.

The wet antenna attenuation coefficients have been derived from observations of six intensive convective rainstorms (Table 1) recorded in central Israel during the winters 2006, 2007 and 2008 by a commercial network of 21 vertically polarized microwave links, operating at frequencies $18-23 \mathrm{GHz}$ with lengths varying from 0.81 to $7.26 \mathrm{~km}$, installed in 12 different locations. The links record quantized instantaneous microwave attenuation with $\Delta=1 \mathrm{~dB}$ magnitude and one minute temporal resolution. For comparison, five rain gauges, recording point rain rate with $6 \mathrm{~mm} \mathrm{~h}^{-1}$ magnitude and one minute temporal resolution, have been installed in the vicinity of microwave links (Fig. 2).

The rest of parameters have been derived using the DSD database consisting of 6282 DSD spectra, collected in central Israel during 1984-1985 (courtesy of Zev Levin; see Feingold and Levin (1986) for details) at the temporal resolution of one minute.

\subsection{Derivation of power law coefficients}

To transform the DSD time series into spatial profiles knowing the rainstorm advection velocity, the Taylor's hypothesis of frozen turbulence is invoked (Leijnse et al., 2008a). As a result, the integration of the space-varying $N_{d}(D, x)$ along the link can be replaced by integration of discrete point-scale DSD time series

$\bar{N}_{d}(D)=\int_{0}^{L} d x N_{d}(D, x) \cong \frac{L}{[L / v]} \sum_{t=1}^{[L / v]} N_{d}(D, t)$,

where $[\cdot]$ stands here for rounding operation, $v$ is the rainstorm advection velocity that has been estimated by correlating multiple microwave links (Zinevich et al., 2009); the (climatological) average for six studied rainstorms (see Table 1) is $v=14.6 \mathrm{~m} \mathrm{~s}^{-1}$. 
Table 1. List of rainstorms, used for empirical assessment of MSE prediction accuracy.

\begin{tabular}{lrrrr}
\hline Event & $\begin{array}{c}\text { Duration, } \\
\mathrm{h}\end{array}$ & $\begin{array}{c}\text { Net rainfall } \\
\text { duration, h }\end{array}$ & $\begin{array}{c}\text { Peak rain } \\
\text { rate, } \mathrm{mm} \mathrm{h}^{-1}\end{array}$ & $\begin{array}{c}\text { Average rain } \\
\text { rate, } \mathrm{mm} \mathrm{h}^{-1}\end{array}$ \\
\hline 26 December 2006 & 22 & 9.6 & 84 & 2.97 \\
5 January 2007 & 62 & 11.6 & 48 & 0.81 \\
29 January 2007 & 38 & 5.7 & 72 & 0.59 \\
4 January 2008 & 9.5 & 1.9 & 54 & 1.22 \\
29 January 2008 & 51 & 7.1 & 54 & 1.48 \\
14 February 2008 & 20 & 2.6 & 42 & 1.09 \\
\hline Total & 202.5 & 38.5 & & 1.22 \\
\hline
\end{tabular}

Table 2. Conventional power-law coefficients $a, b$ for frequency bands in use, determined from $\alpha, \beta$ estimated using Eq. (26) for average link length of $3.43 \mathrm{~km}$.

\begin{tabular}{lrr}
\hline $\begin{array}{l}\text { Frequency } \\
\text { band }\end{array}$ & $a$ & $b$ \\
\hline $18 \mathrm{GHz}$ & 0.069 & 1.154 \\
$19 \mathrm{GHz}$ & 0.070 & 1.145 \\
$22 \mathrm{GHz}$ & 0.099 & 1.121 \\
$23 \mathrm{GHz}$ & 0.110 & 1.112 \\
\hline
\end{tabular}

The parameters $\alpha, \beta$ in Eq. (6) have been obtained using a non-linear fit of $N_{\mathrm{R}}=6200 \mathrm{DSD}$ profiles $\bar{N}_{d}(D)$, using the T-matrix method for extinction cross-section (Mishchenko, 2000):

$[\alpha, \beta]=\underset{\alpha, \beta}{\arg \min } \sum_{i=1}^{N_{R}}\left(R_{L}(i)-\hat{R}_{L}\left(A_{\mathrm{R}}(i)\right)\right)^{2}$,

where $A_{\mathrm{R}}, R_{L}$ and $\hat{R}_{L}$ are given by Eqs. (4), (5) and (6). The problem in Eq. (26) and the rest of non-linear minimization problems in this study are solved using simplex optimization (Press et al., 1992); preliminary coarse grid search has been done to find optimal initial values, likely leading to a global minimum.

Dealing with disdrometer records requires addressing the sampling error issue; it was shown by Uijlenhoet et al. (2006) that the sampling distribution of any DSD moment converges asymptotically to Gaussian with increase of sample size. The sampling distribution of high moments such as rain rate remains skewed for sample size as large as 500 samples, which results in biased estimates of bulk rainfall variables. In the DSD records used in this study, a typical DSD sample size is a few thousand drops for point rain rates of above $1 \mathrm{~mm} \mathrm{~h}^{-1}$ at one minute resolution. For this reason, the effect of the sampling errors on power-law coefficients is assumed to be negligible.

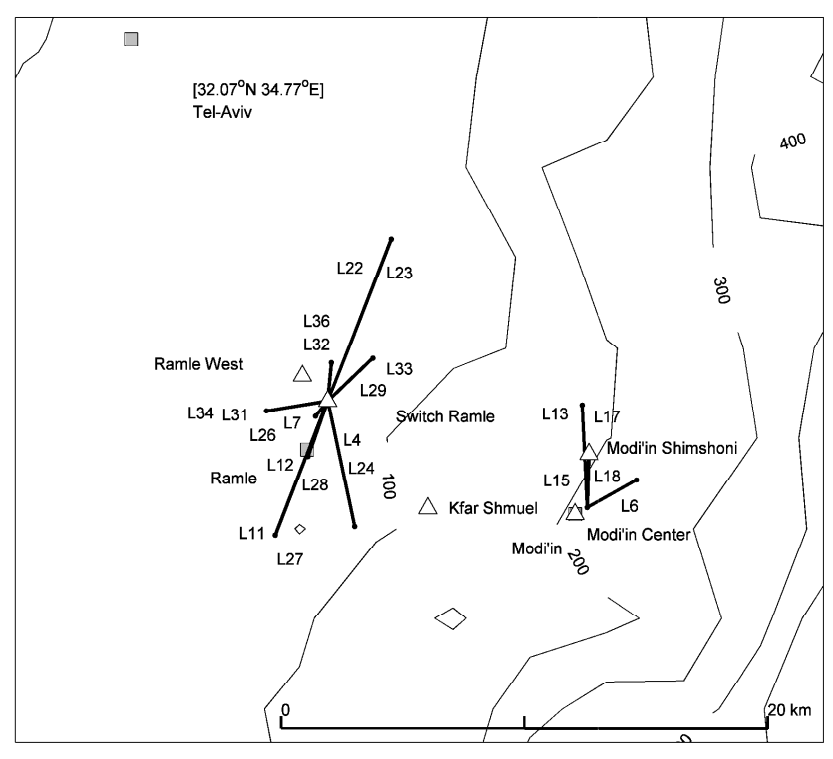

Fig. 2. Locations of microwave links, used for rainfall observations, around the cities of Ramle and Modi'in $(\square)$ and rain gauges $(\Delta)$ Ramle West, Switch Ramle, Kfar Shmuel, Modi'in Shimshoni and Modi' in Center. The local topography contours are given in meters. The duplicating links installed in parallel are denoted twice, e.g. L22 and L23.

The resulting power law coefficients for typical frequency bands are listed in the Table 2. The power-law functions have been found similar to the lognormal model (Zhang and Moayeri, 1999), especially in low rain rates that is in agreement with Feingold and Levin's (1986) conclusion regarding Israeli DSD, even though the actual values of the coefficients differ since non-linear minimization (Leijnse et al., 2007b) has been applied in Eq. (26) versus linear optimization in log domain by Zhang and Moayeri (1999).

\subsection{Derivation of wet antenna attenuation coefficients}

It has been shown by Leijnse et al. (2008a) that wet antenna attenuation is essentially independent on frequency at $17-23 \mathrm{GHz}$, so in this study only link length dependence 
has been assumed for $c_{1}, c_{2}$ (i.e. different coefficients for link length ranges $0 \ldots 1 \mathrm{~km}, 1 \ldots 2 \mathrm{~km}$, etc.). Assuming that nearby gauge rainfall records $\left\langle R_{0}\right\rangle$ averaged over $\Delta t$ minutes approximate averaged link rainfall $\left\langle R_{L}\right\rangle$, the coefficients $c_{1}$, $c_{2}$ are

$c_{1}, c_{2}=\arg \min \sum_{i}\left(\left\langle R_{0}\right\rangle_{i}-\left\langle\hat{R}_{L}\left(\hat{A}_{\mathrm{R}}\right)\right\rangle_{i}\right)^{2} w_{i}$,

$\langle\cdot\rangle_{i}=\sum_{j=i}^{i+\Delta t-1}(\cdot)_{j} / \Delta t$,

where $\hat{A}_{\mathrm{R}}, \hat{R}_{L}\left(\hat{A}_{\mathrm{R}}\right)$ are given by Eqs. (17) and (19); averaging $\langle\cdot\rangle_{i}$ lowers differences due to link-gauge physical separation. Summation through $i$ goes over all available data (Table 1), and the weights $w_{i}$ are chosen to give the same weight to different rainstorm events; otherwise, estimates are biased towards longer events. In practice, estimates of $c_{1}, c_{2}$ are reliable at $\Delta t \geq 10 \mathrm{~min}$ (i.e. $c_{1}, c_{2}$ change weakly with further increase of $\Delta t$ ). Assuming that the sample mean (summation in Eq. 27) approximates the expectation operator, the estimate of $\hat{R}_{L}\left(A_{\mathrm{R}}\right)$ is unbiased (Papoulis, 1991); the bias caused by $n_{\mathrm{w}}$ in Eq. (12) is absorbed into the coefficients $c_{1}, c_{2}$.

Due to the difference in the nature of observations, a single point gauge is not necessarily representative of the link path-averaged rain rate due to spatial rainfall variation. Spatial variations may lead to considerable differences in pathaveraged rainfall amount in the link's location and point rainfall amount at the gauge's location, even though the link and the gauge are installed in close proximity; in this case, the link-gauge difference in rainfall intensities and baseline errors will be absorbed into the wet antenna attenuation coefficients when the latter are calibrated using Eq. (27). However, in the climatological scale, the gauge records can be considered representative of the areal average rain rate; the link-gauge differences in the recorded rainfall amount will decrease with increase of the number of different realizations used for calibration. Eq. (27) requires therefore minimization over much data that comes from multiple links, oriented in various directions. The Fig. 3 demonstrates that for the link length ranges where much data are available (e.g. $2 \ldots 3 \mathrm{~km}$ ), the link-gauge differences in total recorded rainfall amount per event are widely scattered around zero, that indicates that realization-specific differences in rainfall intensity at link-gauge locations have little effect on the resulting wet attenuation coefficients.

The list of the coefficients $c_{1}, c_{2}$ is given in Table 3. One can see that, in general, the coefficient $c_{1}$ that determines maximum (saturation) value of the wet attenuation correction (Eq. 8) lowers for longer links, due to increase of the spatial rain rate variability along longer links and increase of chances that rainfall, captured by a link in the middle, does not affect one or both antennas. The coefficients $c_{1}$ lie close to the range of $3.32-8 \mathrm{~dB}$, reported in the literature by Minda

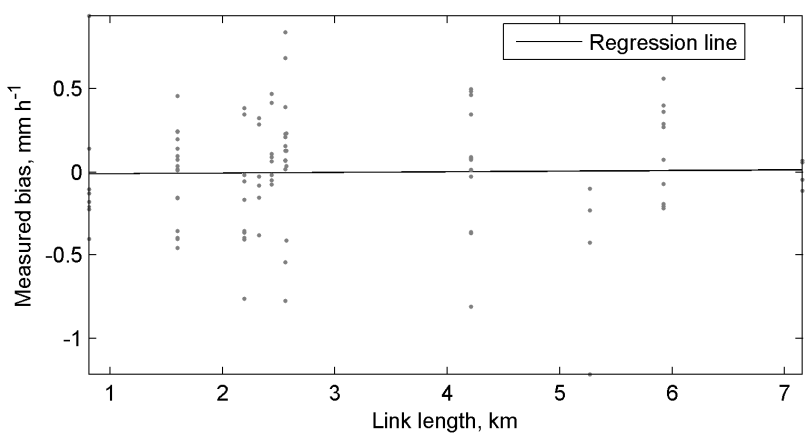

Fig. 3. Differences between total link path-averaged rainfall amount and a nearby rain gauge measurements per link-gauge pair, as a function of link length, over the entire database.

Table 3. Wet antenna attenuation coefficients $c_{1}, c_{2}$ for various link lengths.

\begin{tabular}{ccc}
\hline $\begin{array}{l}\text { Link lengths } \\
\text { range }\end{array}$ & $c_{1}, \mathrm{~dB}$ & $c_{2}, \mathrm{~dB}^{-1}$ \\
\hline $0 \ldots 1 \mathrm{~km}$ & 8.707 & 0.196 \\
$1 \ldots 2 \mathrm{~km}$ & 7.441 & 0.149 \\
$2 \ldots 3 \mathrm{~km}$ & 8.876 & 0.112 \\
$4 \ldots .5 \mathrm{~km}$ & 6.409 & 0.136 \\
$5 \ldots 6 \mathrm{~km}$ & 4.227 & 0.289 \\
$7 \ldots 8 \mathrm{~km}$ & 4.631 & 0.203 \\
\hline
\end{tabular}

and Nakamura (2005), Leijnse et al. (2007b), Kharadly and Ross (2001). Note that the latter reported relatively high $c_{1}=8 \mathrm{~dB}$ by directly measuring wet antenna attenuation, excluding variations of rainfall along a link, that is similar to the case of short links in Table 3.

The records of 9 out of 13 available link-gauge pairs including $7.16 \mathrm{~km}$ links lead the optimization in Eq. (27) to excessively large values of $c_{1}$ (tens of $\mathrm{dB}$ ) due to rainfall intensity variations. The long links, installed roughly orthogonally to the typical rainstorm advection direction capture parts of rainstorm missed by the gauges, located apart. This leads to underestimation of rare high-intensity peaks since the optimization (Eq. 27) concentrates on link-gauge mismatch (rainfall captured by a link but missed by a gauge) in more abundant (low) rain rates. This indicates impossibility of calibration of long links for wet antenna attenuation using Eq. (27) in the present setup due to either high spatial rain rate variations or, possibly, baseline variations that are not represented by pre/post rainstorm measurements. These link-gauge pairs have been excluded from further consideration. As a result, the variation of link-gauge differences for $7.16 \mathrm{~km}$ links is small (Fig. 3) since only similar link-gauge records have been retained. 

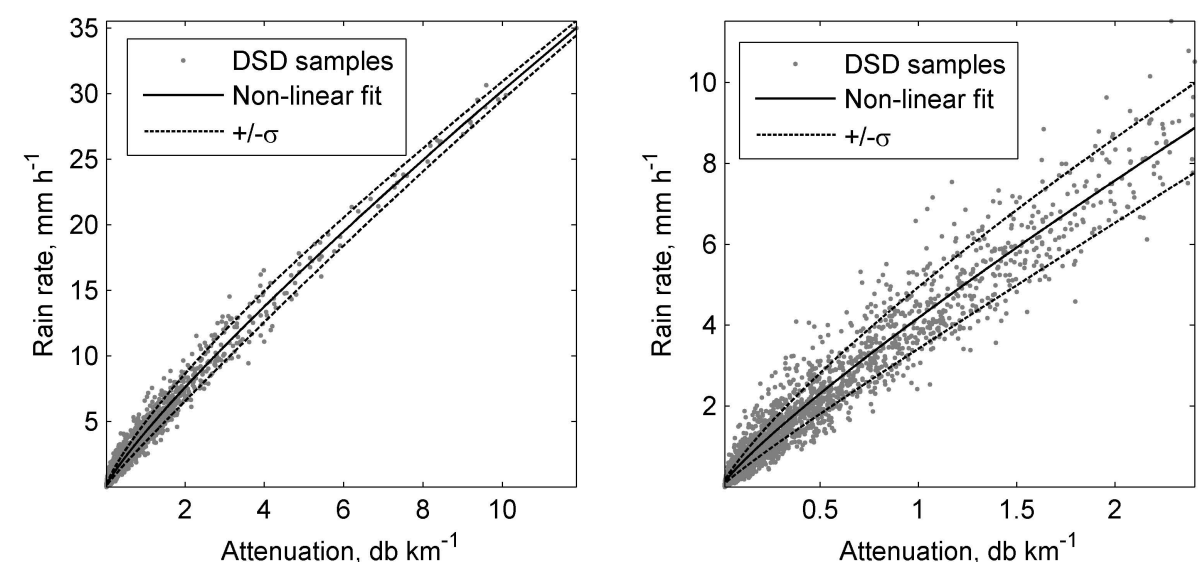

Fig. 4. An example of the power-law fit (Eq. 6) and predicted RMSE (Eq. 11) of path-integrated attenuation (Eq. 4) and path-averaged rain rate (Eq. 5) of the DSD database, including variations in DSD and wet antenna attenuation, for a $4 \mathrm{~km} 20 \mathrm{GHz}$ link. The right figure is a zoomed version of the left one.

Note that the errors in the determination of $\hat{A}_{\mathrm{w}}$ have less impact on the measurement accuracy for longer links since the relative contribution of $A_{\mathrm{w}}$ into the total measured $A_{\mathrm{M}}$ is lower.

\subsection{Derivation of coefficients of path-averaged rainfall MSE model}

Firstly, for each of $N_{\mathrm{R}}$ path-integrated DSD profiles $\bar{N}_{d}(D)$, calculated according to Eq. (25) from the available DSD data, the path-integrated attenuation $A_{\mathrm{R}}$ is computed using Eq. (4). The instantaneous DSD spectra, multiplied by link length $L \cdot N_{d}(D, 1)$ and $L \cdot N_{d}(D,[L / v])$ at two ends of each profile are substituted into Eq. (4) to calculate path-integrated attenuation values $A_{\mathrm{R}}(j), j=1,2$, simulating constant DSD along the link. Then, the wet antenna attenuations for two antennas $A_{\mathrm{w}}(j), j=1,2$ are obtained by solving

$A_{\mathrm{w}}(j)=a_{\mathrm{w}}\left(A_{\mathrm{R}}(j)+A_{\mathrm{w}}(j)\right)$.

Equation (29) is inverted for unknown $A_{\mathrm{w}}(j)$ by golden section search; the value $a_{\mathrm{w}}=\left(A_{\mathrm{w}}(1)+A_{\mathrm{w}}(2)\right) / 2$ is the simulated wet antenna attenuation. Finally, the path-integrated $\hat{A}_{\mathrm{R}}(i)$ is calculated from the full $i$-th DSD profile as

$\hat{A}_{\mathrm{R}}(i)=A_{\mathrm{R}}+A_{\mathrm{w}}-a_{\mathrm{w}}\left(A_{\mathrm{R}}+A_{\mathrm{w}}\right)$.

The above estimation is valid under the assumption that $a_{\mathrm{w}}$ with $c_{1}, c_{2}$ calibrated using Eq. (27) is applicable for the case of constant DSD along the link. With real data, this assumption, in general, does not hold due to non-linearity of $a_{\mathrm{w}}$ :

$E\left[A_{\mathrm{w}}-a_{\mathrm{w}}\left(A_{\mathrm{R}}+A_{\mathrm{w}}\right) \mid A_{\mathrm{w}}\right] \neq 0$.

The bias increases with link length and rain rate. Over the available DSD data, the average bias $\left(\hat{A}_{\mathrm{R}}\right.$ underestimates true $A_{\mathrm{R}}$ ) is about $5 \%$ of $A_{\mathrm{R}}$ (maximum $10 \%$ for high rain rates) for links shorter than $3 \mathrm{~km}$ (two thirds of the studied data) and reaches $7 \%$ (maximum $17 \%$ ) for $7.16 \mathrm{~km}$ links as rainfall variability along the link increases. These results suggest dependence of parameters of the Eq. (8) on rainfall spatial variability; the model, given by Eqs. (29), (30) is better suited for stratiform, homogeneous rainfall, or short links. The optimal wet attenuation coefficients (i.e. producing least biased estimates of rainfall) may therefore be different for different types of rainfall (e.g. convective or stratiform). In general, a more accurate model for wet antenna is needed (e.g. Leijnse et al., 2008a). The latter, however, requires calibration with gauges, installed at both antenna locations for each link that are unavailable. Equations (29), (30) has been used in the present study despite biasedness, assuming that they still allow estimating the typical scale of wet antenna-related errors.

Next, $\hat{R}_{L}\left(\hat{A}_{\mathrm{R}}(i)\right), i=1, \ldots, \mathrm{N}_{R}$ are calculated for all DSD profiles using Eq. (21), and the parameters of the model for MSE of DSD-related uncertainties are calibrated as

$$
\begin{aligned}
{[\gamma, \delta, \varepsilon]=} & \underset{\gamma, \delta, \varepsilon}{\arg \min } \sum_{i=1}^{N_{R}}\left(\left(R_{L}(i)-\hat{R}_{L}\left(\hat{A}_{\mathrm{R}}(i)\right)\right)^{2}\right. \\
& \left.-\hat{\sigma}_{\mathrm{DSD}+\mathrm{Wet}}^{2}\left[R_{L} \mid \hat{A}_{\mathrm{R}}(i)\right]\right)^{2} .
\end{aligned}
$$

As in Eq. (24), the $i$-th sample $R_{L}(i)$ and $\hat{R}_{L}(i)$ are given by Eqs. (5), (6). Examples of $\hat{R}_{L}\left(\hat{A}_{\mathrm{R}}\right)$ and $\hat{\sigma}_{\mathrm{DSD}+\text { Wet }}\left[R_{L} \mid \hat{A}_{\mathrm{R}}\right]$ are shown in Figs. 4, 5. RMSE increases substantially with link length, due to increased variability between antenna locations. Conversely, excluding wet antenna-related variability from consideration (using Eq. 7 instead of Eq. 11) leads to decrease of MSE for longer links (not shown here); for instantaneous measurements, wet antenna effects mostly dominate the effect of DSD variability along a link. Increasing frequency directly leads to accuracy improvement; thus, 
Table 4. The performance statistics $S_{\mathrm{DSD}+\text { Wet }}$ Eq. (33) for various rain rates and frequencies.

\begin{tabular}{rrrrrrrrr}
\hline$R, \mathrm{~mm} \mathrm{~h}^{-1}$ & $2 \mathrm{~km}$ & $2 \mathrm{~km}$ & $2 \mathrm{~km}$ & $2 \mathrm{~km}$ & $0.5 \mathrm{~km}$ & $4 \mathrm{~km}$ & $8 \mathrm{~km}$ & $8 \mathrm{~km}$ \\
& $16 \mathrm{GHz}$ & $20 \mathrm{GHz}$ & $24 \mathrm{GHz}$ & $38 \mathrm{GHz}$ & $20 \mathrm{GHz}$ & $20 \mathrm{GHz}$ & $20 \mathrm{GHz}$ & $38 \mathrm{GHz}$ \\
\hline $1-10$ & 0.99 & 0.99 & 1.00 & 1.14 & 1.00 & 0.99 & 1.00 & 0.98 \\
$10-100$ & 0.96 & 0.97 & 0.98 & 0.96 & 0.99 & 0.97 & 0.99 & 1.04 \\
\hline
\end{tabular}
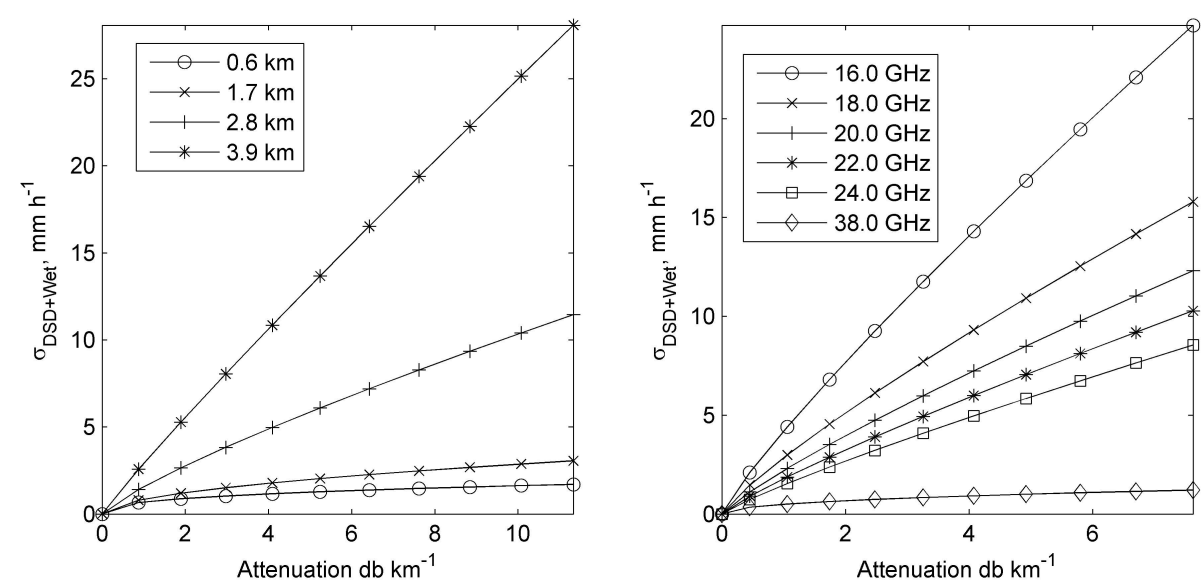

Fig. 5. The predicted RMSE of rain rate estimates $\hat{\sigma}_{\mathrm{DSD}+\mathrm{Wet}}\left(R_{L} \mid A_{\mathrm{R}}+n_{\mathrm{W}}\right)$ (Eq. 11) as a function of link length for a $22 \mathrm{GHz}$ verticallypolarized link (left) and as a function of link frequency for a $2.2 \mathrm{~km}$ link (right).

$18 \mathrm{GHz}$ link is almost twice more uncertain than $24 \mathrm{GHz}$ one (Fig. 5, right). These results are in agreement with conclusions of Atlas and Ulbrich (1977), Leijnse et al. (2008a).

To assess the accuracy of the approximation of $\hat{\sigma}_{\mathrm{DSD}+\text { Wet }}^{2}$ by Eq. (11), the statistics

$S_{\mathrm{DSD}+\text { Wet }}=\sqrt{\frac{\sum_{i=1}^{N_{R}} \hat{\sigma}_{\mathrm{DSD}}^{2}+\text { Wet }\left[R_{L}(i) \mid \hat{A}_{\mathrm{R}}(i)\right]}{\sum_{i=1}^{N_{R}}\left(R_{L}(i)-\hat{R}_{L}\left(\hat{A}_{\mathrm{R}}(i)\right)\right)^{2}}}$.

has been calculated for various frequencies $16 \ldots 38 \mathrm{GHz}$ and link lengths $0.5 \ldots 8 \mathrm{~km}$. Values $S_{\mathrm{DSD}+\text { Wet }}$ close to one indicate validity of Eq. (11). One can see that in most cases, the error does not exceed few percents, with maximum of 14 percent (Table 4).

The model for $\hat{\sigma}_{\mathrm{DSD}}^{2}$ (Eq. 7) can be verified similarly to Eq. (11) using statistics in Eq. (33), producing results, similar to ones in Table 4.

In the case of temporal averaging, the coefficients $\gamma, \delta, \varepsilon$ differ from ones in Eq. (11) as they are calibrated over timeaveraged data to take into account correlation between adjacent time frames

$[\gamma, \delta, \varepsilon]=\underset{\gamma, \delta, \varepsilon}{\arg \min } \sum_{i=1}^{N_{R}}\left(\left(\left\langle R_{L}\right\rangle_{i}-\left\langle\hat{R}_{L}\right\rangle_{i}\right)^{2}-\left\langle\hat{\sigma}_{\mathrm{DSD}+\mathrm{Wet}}^{2}\left[R_{L} \mid \hat{A}_{\mathrm{R}}\right]\right\rangle_{i}\right)^{2}$

where $\langle\cdot\rangle_{i}$ is given by Eq. (28). Accuracy of the model for $\Delta t=1$ and $30 \mathrm{~min}$ is comparable.

\section{Estimation of point rainfall from path-averaged measurements}

To compare path-averaged rainfall with the point scale rain gauges, one can address modeling of rainfall spatial variability through the use of geostatistics methods (Schabenberger and Gotway, 2005) to obtain an MSE expression for rainfall estimation at an arbitrary point in space.

\subsection{Semivariogram modeling}

Under the assumption of stationarity of a two-dimensional rainfall field and its isotropy (covariance between rainfall at two points depends only on distance between them; the validity of this assumption is discussed in the Sect. 5.2), an empirical semivariogram $\gamma(h)$ describes the spatial correlation of rainfall $r$ between two points, separated by distance $h$

$2 \gamma(h)=E\left[\left(r_{\boldsymbol{x}}-r_{\boldsymbol{x}(h)}\right)^{2}\right]$,

where $\boldsymbol{x}(h)=\left\{\boldsymbol{x}^{\prime}:\left\|\boldsymbol{x}-\boldsymbol{x}^{\prime}\right\|=h\right\}$. In practice, an empirical semivariogram $\gamma_{E}(h)$ is firstly calculated from rainfall data by replacing the expectation operator in Eq. (35) by sample mean and then is approximated by Gaussian semivariogram model (subjectively chosen as it fits Eq. (35) best)

$\gamma_{M}(h)=(s-n)\left(1-\exp \left(-\frac{h^{2}}{r^{2}}\right)\right)+n$. 
where sill $s$, nugget $n$ and range $r$ are estimated by non-linear least square fit.

Equation (36) does not assume any a-priori information except for climatologically averaged positive spatial autocorrelation that decreases with distance; in particular, it does not distinguish between weak and strong rainfall. To take this into account, let us assume that the path-averaged rainfall $R_{L}$ represents the local areal average rainfall in the vicinity of a link (e.g. over a circular area with diameter equal to the link length). Consequently, one can consider an optimal estimate of $\gamma(h)$ given $R_{L}$. The conditional semivariogram is defined as

$2 \gamma\left(h \mid R_{L}\right)=E\left[\left(r_{x}-r_{x(h)}\right)^{2} \mid R_{L}\right]$.

Modeling of $\gamma\left(h \mid R_{L}\right)$ has been done in two steps. Firstly, empirical conditional semivariograms have been calculated over a series of $N_{q}$ rainfall intensity ranges $\left\{q_{i}\right\}_{i=1, \ldots, N_{q}}=$ $\left\{\left[0, \ldots, p^{0}\right), \ldots,\left[p^{i-2}, \ldots, p^{i-1}\right), \ldots\right\}$ as

$2 \gamma_{E}\left(h \mid q_{i}\right)=\left\langle\left(r_{\boldsymbol{x}_{1}}-r_{\boldsymbol{x}_{2}}\right)^{2} \mid h, R_{L}\right\rangle$,

where $\langle\cdot\rangle$ denotes averaging over all possible $\left\{\boldsymbol{x}_{1}, \boldsymbol{x}_{2}:\left\|\boldsymbol{x}_{1}-\boldsymbol{x}_{2}\right\|=h, \quad R_{L}\left(\boldsymbol{x}_{1}, \boldsymbol{x}_{2}\right) \in q_{i}\right\}, \quad R_{L}\left(\boldsymbol{x}_{1}, \boldsymbol{x}_{2}\right)$ is the local areal average rainfall intensity in the vicinity of $x_{1}, x_{2}$. The parameter $p=1.5$ has been chosen to maximize $N_{q}$ provided that no rainfall bins $q_{i}$ are empty, given the available rain gauge data. The average rainfall estimate $\left(r_{x_{1}}+r_{x_{2}}\right) / 2$ is substituted for unknown $R_{L}\left(x_{1}, x_{2}\right)$. Then, the models $\gamma_{M}\left(h \mid \bar{R}_{L}(i)\right), \quad i=1, \ldots, \mathrm{N}_{q}$ have been fitted with these empirical semivariograms, producing a set of parameters $\Lambda_{i}=\left\{s_{i}, n_{i}, r_{i}\right\}, i=1, \ldots, N_{q}$

$\Lambda_{i}=\arg \min \sum_{h}\left(\gamma_{E}\left(h \mid q_{i}\right)^{1 / 2}-\gamma_{M}\left(h \mid \bar{R}_{L}(i)\right)^{1 / 2}\right)^{2}$,

where average $\bar{R}_{L}(i)=\left\langle R_{L}\left(\boldsymbol{x}_{1}, \boldsymbol{x}_{2}\right) \in q_{i}\right\rangle$. Taking square root of semivariograms in Eq. (39) is necessary to give more weight to small $h$ (small $\gamma$ ) w.r.t. large $h$ (considerably larger values of $\gamma$ ) in numerical optimization.

The empirical semivariograms have been calculated at three different $h(1.47,6.1$ and $11 \mathrm{~km})$ from the records of four rain gauges over three rainstorms (Fig. 2 and Table 1). In general, it is possible to get the experimental data over a denser range of distances from even single rain gauge record at $1 \mathrm{~min}$ resolution, by invoking the Taylor hypothesis (using climatological average rainfall advection velocity $14.6 \mathrm{~m} \mathrm{~s}^{-1}$ ) and transforming the time series into a spatial profile at the spatial resolution of $0.88 \mathrm{~km}$. To generate temporally averaged data for a $\Delta t$ minutes interval, one should pass the time series via a rectangular moving average filter of $\Delta t$ samples length. However, this operation introduces unrealistic correlation between adjacent samples. To avoid this, one can subsample the filtered time series at $\Delta t$ samples rate, but then the temporal (and, accordingly, spatial) resolution becomes $\Delta t$-dependent. To preserve the consistency between experimental semivariograms for different $\Delta t$, separate realizations using different rain gauge pairs have been used instead of applying Taylor hypothesis.

Special attention has been given to the values of $\gamma_{E}\left(h=0 \mid R_{L}\right)$ that are crucial for stability of optimization in Eq. (39) but there is no field data available. Considerable differences of rain rate due to spatial variability appear already at $h=0.4 \mathrm{~km}$, for $0.81 \mathrm{~km}$ link L7 and Switch Ramle gauge (Sect. 5), that should be modeled by a nonzero nugget. To force the non-zero nugget in Eq. (36), it has been set $\gamma_{E}\left(h=0 \mid R_{L}\right)=\gamma_{E}\left(h=1.47 \mid R_{L}\right)$. An example of semivariogram model $\gamma_{M}\left(h \mid R_{L}\right)$ for 10 min average is drawn in Fig. 6. Note that $\gamma_{E}$ for $R_{L}=19.9 \mathrm{~mm} \mathrm{~h}^{-1}$ exhibits decrease at $h=11 \mathrm{~km}$ that violates an assumption behind the non-decreasing model in Eq. (36); this is attributed to limited amount of available high-intensity data for modeling. As a result, multiple peaks, appearing in a specific event (December 2006, Table 1) express in the model. The values of $\gamma_{M}\left(h \mid R_{L}\right)$ for $R_{L}$ different from $\bar{R}_{L}(i), i=1, \ldots, N_{q}$ are obtained by linear interpolation of the family $\gamma_{M}\left(h \mid \bar{R}_{L}(i)\right)$, and for $R_{L}>\bar{R}_{L}\left(N_{q}\right)$ by means of linear extrapolation.

\subsection{Spatial discretization of a microwave link}

Representation of a link in a discrete form is done by dividing it into a set of $N$ short intervals where the rainfall intensity is assumed to be constant; the length of an interval is chosen $0.5 \mathrm{~km}$ (Goldshtein et al., 2009). The measured pathaveraged rainfall in this model is approximated by averaging point rain rates $R\left(\boldsymbol{x}_{i}\right), i=1, . ., N$

$R_{L}=\alpha\left(a \int d \boldsymbol{x} R(\boldsymbol{x})^{b}\right)^{\beta} \cong \frac{1}{N} \sum_{i=1}^{N} R\left(\boldsymbol{x}_{i}\right)$

where the power law coefficients $a, b, \alpha, \beta$ are taken from Eqs. (1) and (6); the integration is done over all points $\boldsymbol{x}$ along the link. The deviation of $R_{L}$ from the true pathaveraged rainfall for $b \neq 1$ is about a few percents (Atlas and Ulbrich, 1977) and is neglected for MSE estimation.

\subsection{MSE of rainfall estimation}

A trivial estimator of the rainfall at the point $x_{0}$ from a nearby link's measurement is the link's path-averaged rainfall itself, $\hat{R}\left(\boldsymbol{x}_{0}\right)=\hat{R}_{L}\left(\hat{A}_{\mathrm{R}}\right)$. The MSE expression for the estimate of $R\left(x_{0}\right)$ is

$\hat{\sigma}^{2}\left[R\left(x_{0}\right) \mid \hat{A}_{\mathrm{R}}\right]=E\left[\left(R\left(\boldsymbol{x}_{0}\right)-\hat{R}_{L}\left(\hat{A}_{\mathrm{R}}\right)\right)^{2}\right]$.

By denoting the error in estimation of path-averaged rainfall $e=R_{L}-\hat{R}_{L}\left(\hat{A}_{\mathrm{R}}\right)$, substituting Eq. (40) into Eq. (41) and denoting $h_{i j}=\left\|\boldsymbol{x}_{i}-\boldsymbol{x}_{j}\right\|, i, j=0, \ldots, N$, Eq. (41) transforms into 

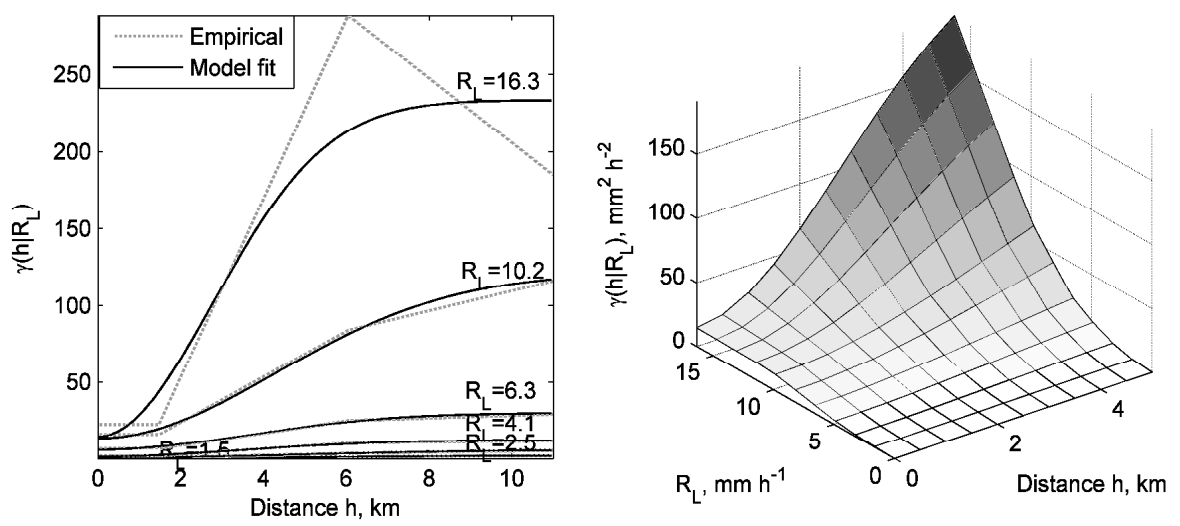

Fig. 6. Examples of empirical and model conditional semivariograms for different values of $R_{L}$ (left) and the resulting three-dimensional function $\gamma\left(h \mid R_{L}\right)$ (right), $10 \mathrm{~min}$ average.

$$
\begin{aligned}
\hat{\sigma}^{2}\left[R\left(x_{0}\right) \mid \hat{A}_{\mathrm{R}}\right] \cong & C(0)+\frac{1}{N^{2}} \sum_{i=1}^{N} \sum_{j=1}^{N} C\left(h_{i j}\right) \\
& -\frac{2}{N} \sum_{i=1}^{N} C\left(h_{i o}\right)+2 E\left[\left(R_{L}-R\left(x_{0}\right)\right) e \mid \hat{A}_{\mathrm{R}}\right]+E\left[e^{2}\right](42)
\end{aligned}
$$

Here $C\left(h_{i j}\right)$ is a covariance function

$C\left(h_{i j}\right)=E\left[R\left(\boldsymbol{x}_{i}\right) R\left(\boldsymbol{x}_{j}\right)\right]-\eta_{i} \eta_{j}$

and $C(0)=\sigma_{R}^{2}$ is a-priori climatological variance of rainfall intensities, under an assumption of constant expected value of rainfall intensity $\eta_{i}=\eta_{j} \equiv \eta \forall \boldsymbol{x}_{i}, \boldsymbol{x}_{j}$ in the area. This assumption is similar to the one of ordinary kriging (Schabenberger and Gotway, 2005); in the climatological scale, the expected rainfall intensity in an area depends on the location (constant per link-gauge pair and over the studied area) and the area size (determined by the link length and the linkgauge distance, constant per link-gauge pair as well).

The term $2 E\left[\left(R_{L}-R\left(x_{0}\right)\right) e \mid \hat{A}_{\mathrm{R}}\right]$ in Eq. (42) describes covariance between $e$ and local rainfall variation $R_{L}-$ $R\left(x_{0}\right)$. While the former is mostly measurement error, the latter is due to difference between path-averaged rainfall and rainfall intensity at a single location $\boldsymbol{x}_{0}$. This term can be neglected under the assumption of independence of $e$ and $R_{L}-R\left(\boldsymbol{x}_{0}\right)$ ( $E[e]$ can be assumed zero according to Eq. 27). However, some components of $e$ (mostly, the errors, related to wet antenna attenuation) do depend on local rainfall variation. Numerical simulation of Eq. (42) using the DSD database (Sect. 3) shows that neglecting $2 E\left[\left(R_{L}-R\left(x_{0}\right)\right) e \mid \hat{A}_{\mathrm{R}}\right]$ in Eq. (42) may lead to errors in $\hat{\sigma}^{2}\left[R\left(\boldsymbol{x}_{0}\right) \mid \hat{A}_{\mathrm{R}}\right]$, depending on $\boldsymbol{x}_{0}$. Thus, locating $\boldsymbol{x}_{0}$ near one of the antennas leads to overestimation of $\hat{\sigma}^{2}\left[R\left(x_{0}\right) \mid \hat{A}_{\mathrm{R}}\right]$ by up to $12 \%$ at $\Delta t=1 \mathrm{~min}$ for long $(7.16 \mathrm{~km}$ links) L22, L23, since the wet antenna-related errors become more severe for longer links; locating $\boldsymbol{x}_{0}$ in the middle of a long link leads to underestimation of $\hat{\sigma}^{2}\left[R\left(x_{0}\right) \mid \hat{A}_{\mathrm{R}}\right]$ by up to $15 \%$ (the correlation between $e$ and $R_{L}-R\left(x_{0}\right)$ is negative in this case). The maximum error, introduced by dropping the term $2 E\left[\left(R_{L}-R\left(x_{0}\right)\right) e \mid \hat{A}_{\mathrm{R}}\right]$, becomes negligible (about $2 \%$ on the average, maximum $4 \%$ ) for $\Delta t$ above $30 \mathrm{~min}$, for all links besides L22, L23.

The calculation of $E\left[\left(R_{L}-R\left(x_{0}\right)\right) e \mid \hat{A}_{\mathrm{R}}\right]$ is complicated since the models of $R_{L}-R\left(x_{0}\right)$ and $e$ are calibrated using different datasets - the point gauge records (Table 1) and the DSD database (Sect. 3), respectively; the dependence of $E\left[\left(R_{L}-R\left(x_{0}\right)\right) e \mid \hat{A}_{\mathrm{R}}\right]$ on $\boldsymbol{x}_{0}$ requires development of an additional model. In this study, we neglect this covariance term, keeping in mind the consequences - overestimation of $\hat{\sigma}^{2}\left[R\left(\boldsymbol{x}_{0}\right) \mid \hat{A}_{\mathrm{R}}\right]$ at short temporal averaging intervals ( $\Delta t$ less than $30 \mathrm{~min}$ ) and for long links L22, L23.

Substituting into Eq. (42)

$C\left(h_{i j}\right)=C(0)-\gamma\left(h_{i j}\right)$

and Eq. (11) for $E\left[e^{2}\right]$, we get the MSE expression in terms of semivariogram

$$
\begin{aligned}
\hat{\sigma}^{2}\left[R\left(\boldsymbol{x}_{0}\right) \mid \hat{A}_{\mathrm{R}}\right] \cong & \frac{2}{N} \sum_{i=1}^{N} \gamma\left(h_{i 0}\right) \\
& -\frac{1}{N^{2}} \sum_{i=1}^{N} \sum_{j=1}^{N} \gamma\left(h_{i j}\right)+\hat{\sigma}^{2}\left[R_{L} \mid \hat{A}_{\mathrm{R}}\right] .
\end{aligned}
$$

In the case of the conditional semivariogram, $\gamma\left(h \mid R_{0}\right)$ is directly substituted into Eq. (45) instead of $\gamma(h)$; this can be done since the condition

$C\left(h_{i j} \mid R_{0}\right)=C\left(0 \mid R_{0}\right)-\gamma\left(h_{i j} \mid R_{0}\right)$

holds under the assumption of constant mean rainfall $R_{0}$ in the vicinity of the link, i.e. $E\left[R\left(x_{j}\right) \mid R_{0}\right]=E\left[R\left(x_{i}\right) \mid R_{0}\right]=$ $R_{0}$ for all pairs of $i, j=0, \ldots, \mathrm{N}$. 


\subsection{Verification of the spatial uncertainty model}

To verify the proposed semivariogram-based spatial uncertainty model, one can conduct a self-consistency check, similar to the one in Sect. 3.3, using gauge point records.

Since we are interested in evaluation of the model at gauge separation distances different from the ones defined by actual rain gauge installations, used in the Sect. 4.1 for building of the semivariogram model, let us transform the point rain gauge measurement into spatial profiles according to the Taylor hypothesis using climatological average rainstorm advection velocity $\left(14.6 \mathrm{~m} \mathrm{~s}^{-1}\right)$. Then the rain gauge samples at $1 \mathrm{~min}$ temporal resolution can be considered as spatially distinct point measurements at separations $h$ of $0.88,1.75$, $2.64, \ldots, 10.51 \mathrm{~km}$ between the virtual locations $\boldsymbol{x}_{0}, \boldsymbol{x}_{1}$. Note that simulation of accumulation intervals longer than $1 \mathrm{~min}$ becomes complicated, since application of a moving average filter to the rain gauge time series introduces unrealistic correlation between adjacent samples.

The estimate of MSE $\hat{\sigma}^{2}\left[R\left(x_{0}\right) \mid R\left(x_{1}\right)\right]$ in the location $\boldsymbol{x}_{0}$ from a point measurement at $\boldsymbol{x}_{1}$ can be obtained from Eq. (45) by setting $N=1$ and dropping the term $\hat{\sigma}^{2}\left[R_{L} \mid \hat{A}_{\mathrm{R}}\right]$, related to the link measurement uncertainty. The spatial variability prediction statistics $S_{\mathrm{sp}}(h, i)$ is then calculated per each rainfall intensity range $q_{i}, i=1, \ldots, N_{q}$ and each separation distance $h$ as

$$
\begin{aligned}
p(h, i) & =\left\langle\hat{\sigma}^{2}\left[R\left(\boldsymbol{x}_{0}\right)_{j} \mid R\left(\boldsymbol{x}_{1}\right)_{j}\right]\right\rangle_{j(h, i)} \\
& \cong\left\langle 2 \gamma\left(h_{10} \mid \hat{R}_{\mathrm{av} j}\right)-\gamma\left(h_{11} \mid \hat{R}_{\mathrm{av}_{j}}\right)\right\rangle_{j(h, i)}
\end{aligned}
$$

$e(h, i)=\left\langle\left(R\left(x_{0}\right)_{j}-R\left(x_{1}\right)_{j}\right)^{2}\right\rangle_{j(h, i)}$,

$S_{\mathrm{sp}}(h, i)=\sqrt{p(h, i) / e(h, i)}$

where $\hat{R}_{\mathrm{av} j}=\left(\hat{R}\left(\boldsymbol{x}_{0}\right)_{j}+\hat{R}\left(\boldsymbol{x}_{1}\right)_{j}\right) / 2$, similarly to the Sect. 4.1, and $p(h, i)$ and $e(h, i)$ are the average predicted and measured errors in the rainfall intensity range $q_{i}, i=$ $1, \ldots, N_{q}$ and at gauge separation $h$. The summation over the index $j(h, i)$ goes through all available rain gauge data at the separation $h$ and in the range $q_{i}$.

The distribution of $S_{\mathrm{sp}}(h, i)$ is plotted in the Fig. 7. The graph shows that the accuracy lowers (statistics increases up to 1.25 and higher) at high rainfall intensities (where only a few data samples available) and at low rainfall intensity levels. In case of weak rainfall, the rain gauge signal quantization $\left(6 \mathrm{~mm} \mathrm{~h}^{-1}\right)$ leads to non-linear signal distortions that possibly results in the mismatch between predicted and measured errors. At the moderate rain rates of $5-10 \mathrm{~mm} \mathrm{~h}^{-1}$, the model shows statistics $S_{\mathrm{sp}}(h, i)$ close to one, indicating accurate prediction of the variability.

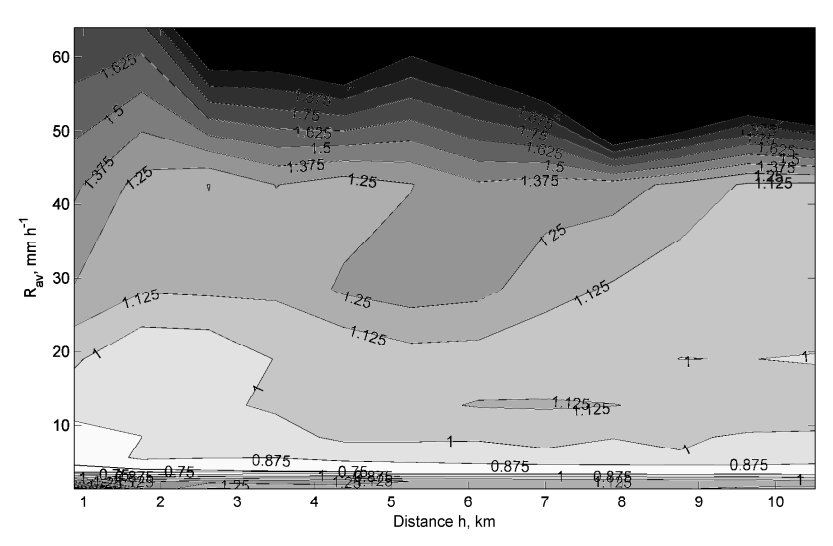

Fig. 7. The spatial variability prediction statistics $S_{\mathrm{sp}}(h, i)$ (Eq. 48), as a function of gauge separation distance $h=0.88,1.75,2.64, \ldots$, $10.51 \mathrm{~km}$, at rainfall intensity ranges $q_{i}, i=1, \ldots, N_{q}, \Delta t=1 \mathrm{~min}$.

An estimate of the expected $\bar{S}_{\mathrm{sp}}$ over the entire database is

$\bar{S}_{\mathrm{sp}}=\sqrt{\sum_{h, i} p(h, i) N(h, i) / \sum_{h, i} e(h, i) N(h, i)}$,

where $N(h, i)$ is the number of samples, falling into the range $q_{i}$ at separation $h$ can give a quantitative estimate of the modeling accuracy for the given experimental setup. Here, $\bar{S}_{\mathrm{sp}}$ equals to 1.04 that indicates a small overestimation of the spatial variability-related errors.

\section{Results and discussion}

Performance of the uncertainty quantification models have been evaluated using records of 96 link-gauge pairs (Sect. 3, Fig. 2) over three convective rainstorms (Table 1). The statistics $S_{j}$ for the accuracy of MSE prediction $p_{j}$ (Xu and Wilke, 2005) w.r.t. measured error $e_{j}(j=1 \ldots 96)$ are

$e_{j}=\sqrt{\frac{1}{T_{j}} \sum_{t=1}^{T_{j}}(R(t, j)-\hat{R}(t, j))^{2}}, \quad p_{j}=\sqrt{\frac{1}{T_{j}} \sum_{t=1}^{T_{j}} \hat{\sigma}_{t j}^{2}}$,

$S_{j}=p_{j} / e_{j}$,

where $t=1, \ldots, T_{j}$ is the index of a sample (averaging over $\Delta t$ minute interval) for the $j$-th link-gauge pair, $R(t, j)$ and $\hat{R}(t, j)$ are the gauge measurement and link estimate at time $t$, and $\hat{\sigma}_{t j}^{2}$ is the predicted MSE, given by Eq. (45). The values of $S_{j}$ close to one indicate correct prediction of measurement errors. To examine relative role of each one of the error sources, the results have been calculated at various temporal averaging intervals $(\Delta t=1,5,10,15,30,60$ and $120 \mathrm{~min})$.

To get insight into the respective contribution of each component of the measured attenuation model into the predicted error, statistics in Eq. (50) have been computed excluding some error sources (i.e. zeroing their respective MSE estimates in Eq. 45): 
1. Spatial+Link - prediction of the total error, including all error sources.

2. Spatial - prediction of error in rainfall estimation at the rain gauge location due to spatial rainfall variability in the link's neighborhood only, assuming link measurements are perfect.

3. $D S D$ - prediction of path-averaged rainfall measurement error due to DSD variability along the link only.

4. Wet - prediction of error due to antenna wetting only.

5. Quant-prediction of quantization error only.

6. Baseline - prediction of baseline-related errors only.

An example time series of the measured error $e_{j}$ vs. predicted RMSE $p_{j}$ for 10-min average rainfall is shown in Fig. 8, bottom. For clarity, the results are presented in the form of measured and predicted RMSE of accumulated rainfall estimates. One can see that at about 4 January 2008, 13:30 LT the link overestimates rainfall w.r.t. Switch Ramle rain gauge, while at about 18:30 the gauge records a strong peak, partially missed by the link (Fig. 8, top). As a result, the error prediction Spatial+Link, based on the link measurements and closely following the measured error until 13:30, overestimates measurement error between 13:30 and 18:30 and underestimates starting from about 18:30 (Fig. 8, bottom). This shows that even at short spatial distance (link length $0.81 \mathrm{~km}$ and the link-gauge distance is $0.41 \mathrm{~km}$ ), spatial rainfall variability strongly affects the error prediction accuracy, and even a single peak may cause considerable measurement errors.

Taking into account this dominating effect of spatial variability and simplifying assumptions made in Sect. 4 (e.g. inferring local areal-average rainfall from link measurements, semivariogram modeling with gauge records), one should expect that the error predictions should be correct only on the average. The total statistics $\bar{S}$ is used to estimate the accuracy of MSE prediction:

$\bar{e}=\sqrt{\sum_{j \in J} \sum_{t=1}^{T_{j}} \hat{\sigma}_{t j}^{2}}, \quad \bar{p}=\sqrt{\sum_{j \in J} \sum_{t=1} T_{j}(R(t, j)-\hat{R}(t, j))^{2}}, \bar{S}=\bar{p} / \bar{e}$

where $J$ is a chosen subset of link-gauge pairs to represent a specific interval of link lengths or rain rates.

\subsection{Accuracy of error predictions at various temporal resolutions}

Figure 9 shows the performance statistics $\bar{S}$ for various error sources at different temporal resolutions (accumulation intervals) $\Delta t$. At all temporal averaging intervals, spatial variability uncertainty dominates the link-related uncertainties (Fig. 9, bottom), even though the role of the latter increases with $\Delta t$. The baseline uncertainty is the major error source
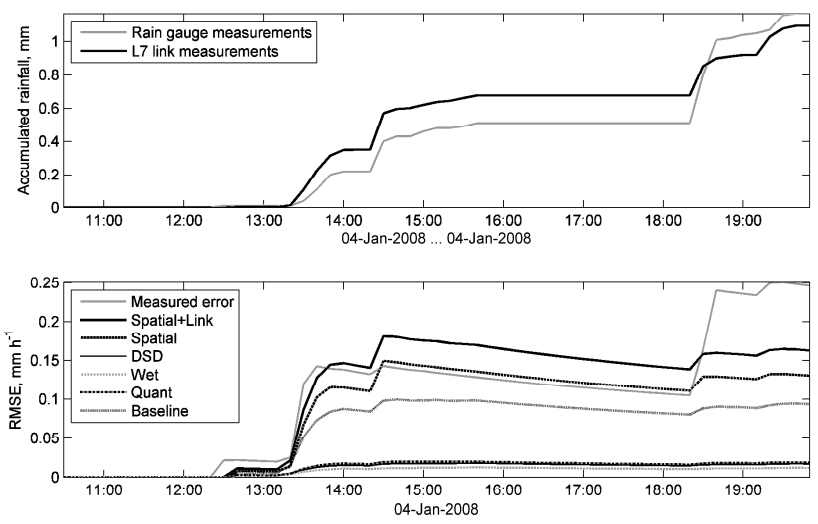

Fig. 8. Time series of accumulated gauge rainfall vs. microwave rainfall (upper plot) and measured vs. predicted RMSE (Eq. 50) of accumulated microwave rainfall estimates (lower plot). Contribution of various error sources into the total predicted error is shown in the lower plot, for L7 link and Switch Ramle gauge in 3 January 2008 rainstorm. Link length is $0.81 \mathrm{~km}$, frequency is $23.27 \mathrm{GHz}$, $\Delta t=10 \mathrm{~min}$. Note the different scales of the upper and lower plots.
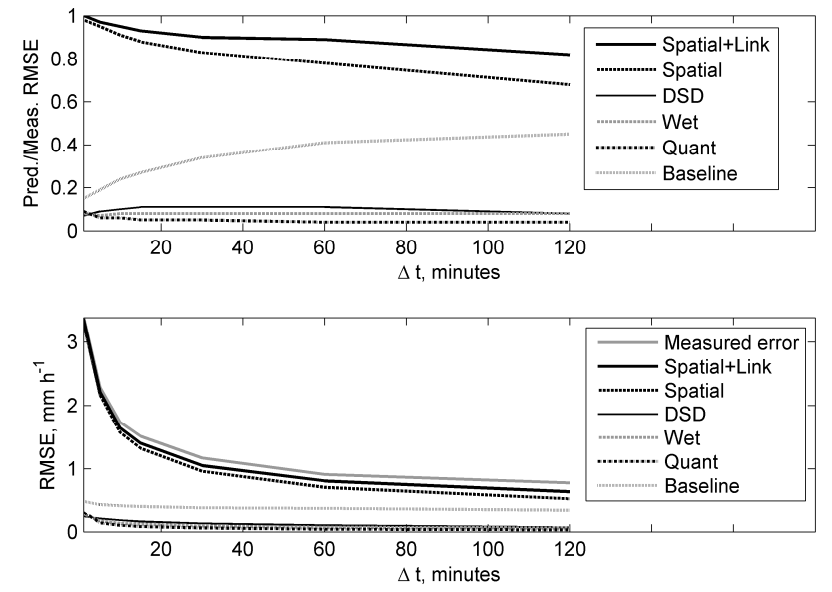

Fig. 9. Performance statistics $\bar{S}$ (top), measured $\bar{e}$ and predicted accumulated errors $\bar{p}$ (Eq. 51) for various error sources (bottom), as a function of temporal averaging interval.

among link-related ones. The predicted wet antenna-related errors decrease with increasing $\Delta t: \bar{p}$ (Wet) changes from $0.28 \mathrm{~mm} \mathrm{~h}^{-1}$ at one minute resolution up to $0.06 \mathrm{~mm} \mathrm{~h}^{-1}$ at $120 \mathrm{~min}$ (Fig. 9, bottom). The predicted quantization errors, independent for different observations, also lower with increasing $\Delta t$ (from 0.31 to $0.03 \mathrm{~mm} \mathrm{~h}^{-1}$ ). The DSD-related errors $\bar{p}(D S D)$ exhibit similar dependence, but to less extent (from 0.24 to $0.07 \mathrm{~mm} \mathrm{~h}^{-1}$ ), due to inter-storm variability in the DSD. 

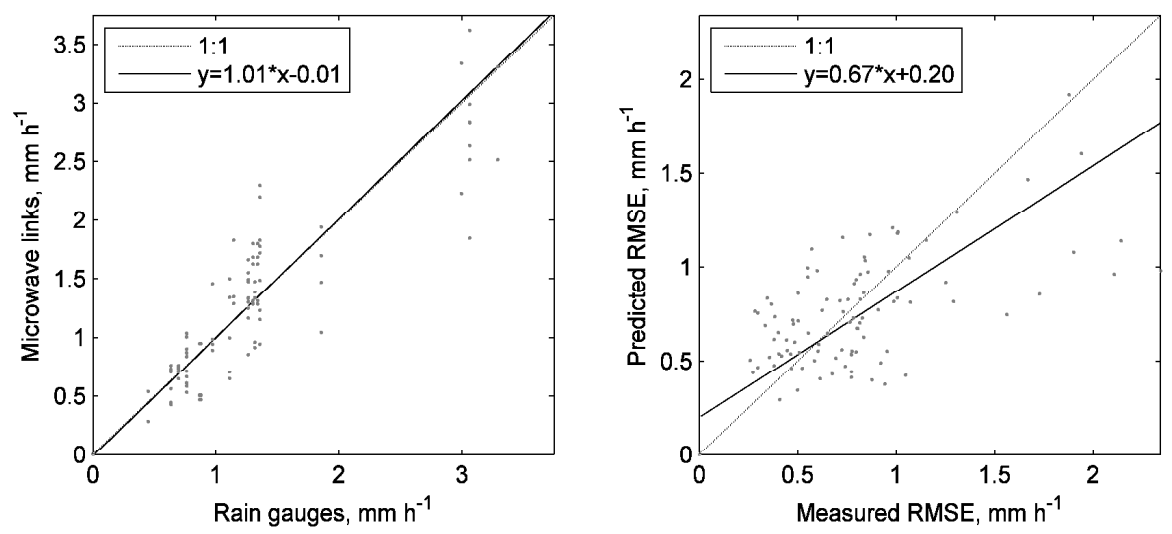

Fig. 10. Scatter plots of the total microwave measured rainfall vs. rain gauge measurements (left) and Spatial + Link estimates of the prediction error vs. measured error (right) for all link-gauge combinations, $\Delta t=60 \mathrm{~min}$. The method of total least squares, assuming uncertainties in both independent and dependent variables (Krystek and Anton, 2007) has been used to draw regression lines.

The statistics $\bar{S}$ varies with $\Delta t$ from 1.00 to 0.82 (Fig. 9, top) that shows more accurate error prediction than it has been reported in the literature (the simulation by Leijnse et al. (2008a) has allowed prediction of $32 \%$ normalized RMSE vs. $94 \%$ measured one, that equals $\bar{S}=0.34$ ), due to inclusion of uncertainties, related to the difference in link-gauge physical locations and baseline estimation uncertainty.

Note that the semivariograms, calculated from the records of two point rain gauges, absorb $6 \mathrm{~mm} \mathrm{~h}^{-1}$ quantization rain gauges errors twice, while in link-gauge comparison it presents only once. This modeling error as well as $\bar{S}$ modeling errors (see Sect. 4.4; the validation statistics $\bar{S}_{\mathrm{sp}}$ (Eq. 49) equals to 1.04) may mask underestimation, leading to $\bar{S}=1.00$ at $\Delta t=1 \mathrm{~min}$. For longer $\Delta t$, this effect quickly diminishes (only amount of water in a bucket before and after the accumulation interval is uncertain). For most link-gauge pairs, the gauge is located at one of the link ends (Fig. 2); this also leads to overestimation in MSE prediction at short $\Delta t$ (less than $30 \mathrm{~min}$ ) due to the neglected covariance term in Eq. (42). The approximation of a link by its midpoint (i.e. setting $N_{w}=1$ in Eq. 40) leads to RMSE overestimation: $\bar{S}$ values reach 1.05 at $\Delta t=1$ min that justifies modeling rainfall variability along a link according to Eq. (42).

The scatter plot (Fig. 10, left) shows that the rainfall estimation is overall unbiased (the regression line is close to 1:1). There are a few groups of points in the graph, corresponding to different events (Table 1); microwave links slightly overestimate gauges for moderate rainstorms (around average gauge rainfall of $1.3 \mathrm{~mm} \mathrm{~h}^{-1}$ ), but underestimate for strong December, 2006 rainstorm (average gauge rainfall or around $3 \mathrm{~mm} \mathrm{~h}^{-1}$ ); a detailed this effect is analyzed in Sect. 5.2. This expresses also in the error comparison scatter plot (Fig. 10, right): high measurement errors (around measured RMSE of $2 \mathrm{~mm} \mathrm{~h}^{-1}$ ) tend to be underestimated at the accumulation interval $60 \mathrm{~min}$. The effect becomes prominent with increase of $\Delta t$ to $120 \mathrm{~min}$, leading to lowering of the regression slope coefficient from 0.77 to 0.52 . For $\Delta t=1$, the regression equation is close to ideal $(y=0.94 x-0.19)$; the slope parameter lower than 1 is compensated by a small positive intersect parameter. At other temporal accumulation intervals the accuracy of error prediction gradually lowers (Fig. 9, top). Thus, for $\Delta t=5 \mathrm{~min}$, the regression equation is $y=0.91 x+0.13$ and the overall bias remains small, $\bar{S}=0.97 \ldots 1.02$. For $\Delta t=10 \ldots 30 \mathrm{~min}$, the slope parameter lowers from 0.92 to 0.72 , and $\bar{S}$ changes from 0.95 to 0.90 (Fig. 9). The degradation of the prediction accuracy with increase of $\Delta t$ is analyzed below for $\Delta t=60 \mathrm{~min}$.

\subsection{Accuracy of error predictions as a function of link length and rain rate}

The dependence of the performance statistics $\bar{S}$ for major error sources as well as measured and predicted errors as a function of link length are shown in the Fig. 11. The spatial variability errors increase with link length; their relative contribution also increases. In most cases, lengths are correlative with link-gauge separation distances (Fig. 2), that contributes as well. Quantization and baseline-related errors behave inversely: for longer links, their contribution lowers. The DSD- and wet antenna- related errors increase for longer links but for different reasons: wet antenna-related errors naturally grow with link length due to increased spatial variability (Fig. 5, left), while the errors due to DSD variability along the link also grow due to lowering frequency band (from $22 \mathrm{GHz}$ for short links to $18 \mathrm{GHz}$ for $4.21-5.92 \mathrm{~km}$ links L11/ L27, L4/L24, L13/L17).

The regression line in Fig. 11 (top left) shows general overestimation of $S_{j}$ for long links; thus, 20 link-gauge pairs with links longer than $4 \mathrm{~km}$ overestimate the predicted errors, versus 10 link-gauge pairs in the same length range showing $S_{j}$ less than 1 . One of the reasons for the overestimation is the neglected covariance term in Eq. (42); another 

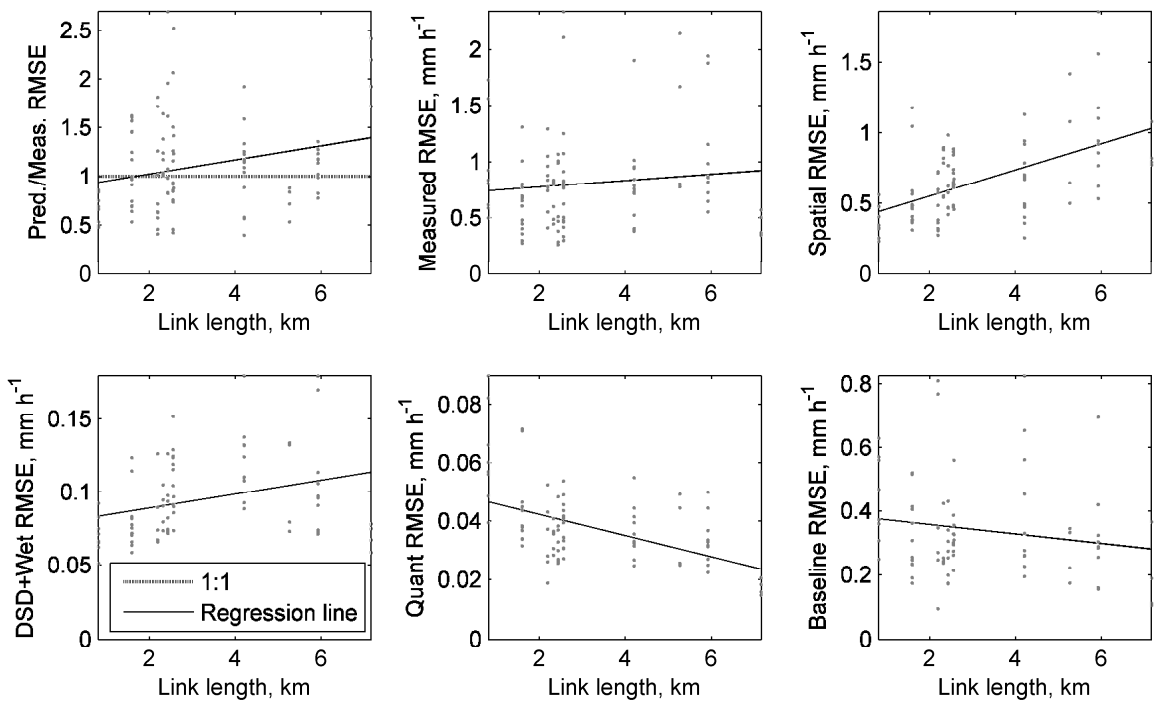

Fig. 11. Performance statistics $S_{j}, j=1, . ., 96$ (Eq. 50) for total predicted error, measured errors and various error sources (spatial variability, DSD and wet antenna, quantization and baseline), as a function of link length, $\Delta t=60 \mathrm{~min}$.

one is the suboptimality of isotropic semivariogram models, discussed next. In general, Israeli convective rainstorms exhibit sequences of fronts driven by western winds (Zinevich et al., 2009), in parallel to the long links L22/L23, L11/L27, L4/L24, L13/L17 (Fig. 2); the variability of rainfall along the front is much lower than predicted. The empirical semivariograms in Eq. (38) have been calculated at $h=6 \mathrm{~km}$ from the records of Switch Ramle and Kfar Shmuel gauges; the semivariograms therefore model the variability roughly orthogonally to the fronts that is much higher. In this case, the assumption behind the isotropic semivariogram has been violated. Note also that only few records from $7.16 \mathrm{~km}$ links where the link-gauge differences are minimal have been chosen (see Sect. 3.2); this is an additional reason for error overestimation. Apparently, these effects overcome the increase of measurement errors for high rain rates for longer links due to suboptimal wet attenuation coefficients (Sect. 3.2).

On the other hand, the errors are underestimated for $0.81 \mathrm{~km}$ links L7, L26, most likely because there are no data available to accurately estimate semivariogram at short gauge separation (that is, non-zero nugget is underestimated in Eq. 36). Note that the effects of overestimation in error prediction for long links remain consistent across all accumulated intervals between 1 to $120 \mathrm{~min}$.

The dependences of $\bar{S}, \bar{e}$ and $\bar{p}$ on average rainfall intensity (as recorded by gauges) are shown in Fig. 12. The contribution of all error sources increases with rain rate, but the growth of spatial variability errors is most prominent. The general trend of RMSE underestimation for the strong rain storm 26 December 2006 (Fig. 12, top left) appears at $\Delta t=15 \mathrm{~min}$ and longer, for six (L12, L7, L4/L24, L11, L31) out of seven link-gauge pairs including Switch Ramle gauge record (average rain rate of $3.06 \mathrm{~mm} \mathrm{~h}^{-1}$ ). The RMSE underestimation follows from rain rate underestimation by links with respect to the gauge. All six links have shown negative bias: $9 \%$ for $0.81-2.56 \mathrm{~km}$ links L12, L7 and L31, and $17 \%$ for 5.26-5.92 km links L4/L24 and L11, which may be either rainfall overestimation by the gauge or underestimation by the links due to high spatial rainfall variability, characterizing this extremely intense event. Comparison of these links with another nearby rain gauge Ramle West (not shown here) demonstrates a similar trend which suggests that it is the links that underestimate rather than the gauge that overestimates. As it has been shown in Sect. 3.3, optimal wet antenna attenuation coefficients lower for intensive highlyvariable events; use of climatologically averaged wet antenna attenuation coefficients leads to underestimation of rain rates for these intensive highly-variable events and overestimation of more homogeneous and uniform rainfall. One can suggest that the prediction of uncertainties due to DSD variations along a link (specifically, antenna wetting, see Sect. 3.3) is not accurate enough for highly intense convective rainstorms (or, possibly, the DSD data used for modeling is not representative for the 26 December 2006 rain storm). On the other hand, at $\Delta t=1 \mathrm{~min}$, a similar trend (decrease of error prediction accuracy with increase of rainfall intensity) arises from overestimation of measured errors for the weakest event 29 January 2007, characterized by very few peaks, highly correlated between rain links and gauges and therefore does not exhibit considerable spatial variability (not shown here). Accordingly, similar trends are observed for other temporal accumulation intervals. 

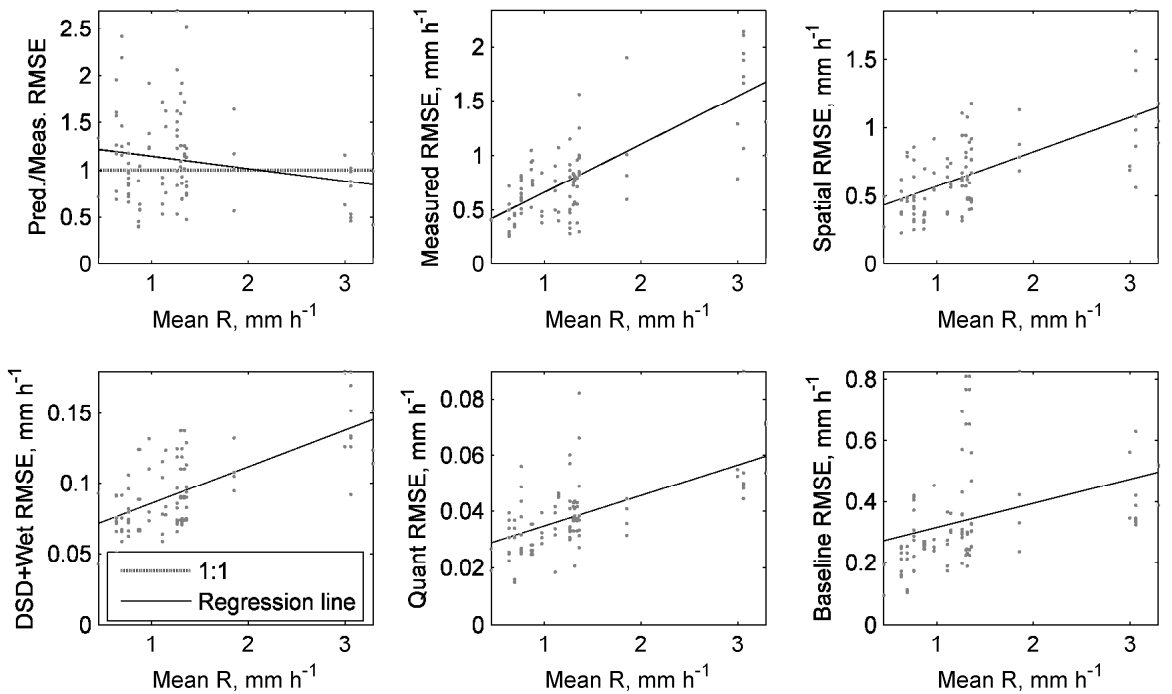

Fig. 12. Performance statistics $S_{j}, j=1, . ., 96$ (Eq. 50) for total predicted error, measured errors and various errors sources (spatial variability, DSD and wet antenna, quantization and baseline), as a function of rain rate recorded by a nearby rain gauge, $\Delta t=60 \mathrm{~min}$.

\section{Conclusions}

Various error sources affecting accuracy of rain rate estimation using commercial microwave links have been examined, and an analytical expression for MSE of rainfall estimation from attenuation, measured by a single link has been derived. Even though a number of simplifying assumptions have been made (e.g. isotropy of the semivariograms and covariance functions, second-order stationarity of distribution of rainfall intensities in space and time), the experimental errors are mostly in agreement with the predicted ones for various link lengths, rain rates and temporal averaging intervals. The accuracy of the link-gauge error prediction is higher than that reported in the literature (Leijnse et al., 2008a), since additional error sources (baseline variability and spatial rainfall variability) have been taken into account in the proposed model. On the other hand, the considered dataset is limited and verification of most of the presented models shows errors of approximately $5-20 \%$, so that the quantification made is not very accurate; however, it still allows understanding typical magnitude and relative contribution of various error sources.

The major source of errors in estimating path-averaged rainfall by a link is the baseline uncertainty that dominates other instrumental (quantization error) and environmental (DSD variability along a link) effects; use of climatologically average wet antenna attenuation coefficients may serve as an additional source of errors. It is known that DSD variability is the major error source in radar backscattering measurements; its effect on forward scattering and absorption measurements by a link is much smaller (Jameson, 1991) and is masked by other error sources. For this reason, the accuracy of prediction of DSD variability-related errors can- not be comprehensively assessed in the presence of other error sources but only based on the point DSD records; it has been shown that its accuracy is likely insufficient for an extremely intense rainstorm. Spatial rainfall variability is the primary source of discrepancy between link-gauge measurements, suggesting that effect of spatial variability will remain major in extrapolation of path-averaged observations into areal averages.

The error calculation has been validated over only six convective rainstorms in Israeli climate; studying stratiform and other types of rainfall is desirable as it may reveal a different relative contribution of the error sources and prediction accuracy. The rest of discrepancies is likely to arise from modeling errors (e.g. a number of simplifying assumptions have been made for spatial variability modeling) and other unaccounted error sources - for example, effects of natural temperature variations (Leijnse et al., 2007a) and anomalous propagation (ducting). It is assumed that the baseline variation during the rainstorm can be adequately described by pre- and post- rainstorm measurements, while plausibility of this assumption is verified only indirectly. On the other hand, some assumptions (e.g. adequacy of quantization error model in Eq. (3), effect of quantization on accuracy of baseline variance estimation, suboptimality of wet antenna-related error model in Eq. (31) and wet attenuation calibration errors) cannot be thoroughly examined in the present setup, since these error sources are minor and are masked by others.

In addition, the presented results are based on an assumption that the wet antenna coefficients and semivariogram models are known perfectly: they have been estimated from link-gauge records over the same events, used subsequently for evaluation. A direct drawback of such approach 
is overfitting: the wet attenuation coefficients may have absorbed a part of other link-gauge differences (e.g. baseline errors, differences due to spatial variability and errors due to inappropriateness of power law coefficients) that in turn may result in overestimation of error prediction accuracy. It has been shown that for long $(7.16 \mathrm{~km})$ links calibration of wet antenna attenuation model with a single rain gauge may be complicated for convective rainstorms; to overcome this, accurate tracking of baseline along the rainstorm and filtering out parts of an event with low link-gauge correlation may be needed. It is assumed that for short links these effects are limited because of large amount of calibration data and various link-gauge combinations. It has been shown that the wet attenuation model is not invariant to the differences in spatial rainfall variability that requires further research.

For practical real-time applications, either climatologically average or forecasted semivariogram models should be used; the baseline attenuation should also be predicted from past dry estimates, and forecast-related errors should be studied as well. In addition, high temporal resolution measurements are not always available; other temporal sampling strategies lead to additional errors (Leijnse et al., 2008a). The MSE expressions for path-averaged rainfall assume specific climatology (the ad hoc parametric model of $\hat{\sigma}_{\text {DSD }}^{2}$ in Eq. (7) has been built according to Israeli DSD data).

The MSE expressions for path-integrated rainfall measurements can further be used in data assimilation algorithms (e.g. Grum et al., 2005; Zinevich et al., 2009) as variance estimates (it has been shown that the bias of rainfall estimation is overall very limited), providing means for weighing observations according to their uncertainty. Similarly, since spatial variability is a major error source, its modeling is essential for reconstruction of spatial rainfall distribution from multiple links. The isotropic semivariogram model allows explaining most of the errors; the experimental results suggest that an anisotropic model would allow higher error prediction accuracy for Israeli convective rainstorms.

Acknowledgements. The authors deeply thank A. Shilo, E. Moshayov (Pelephone) and A. Rayitsfeld (Tel Aviv University) for their cooperation in supplying microwave data and help in installation of rain gauges, and Zev Levin for providing disdrometer data. This research was supported by the ISRAEL SCIENCE FOUNDATION (grant No. 173/08) and the Porter School of Environmental Studies at Tel Aviv University.

Edited by: R. Sussmann

\section{References}

Atlas, D. and Ulbrich, C.: Path- and area-integrated rainfall measurement by microwave attenuation in the $1-3 \mathrm{~cm}$ band, J. Appl. Meteorol., 16, 1322-1331, 1977.

Aydin, K., and Daisley, S.: Relationships between rainfall rate and $35-\mathrm{GHz}$ attenuation and differential attenuation: modeling the effects of raindrop size distribution, canting, and oscillation, IEEE T. Geosci. Remote, 40(11), 2343-2352, 2002.

Berne, A. and Uijlenhoet, R.: Path-averaged rainfall estimation using microwave links: Uncertainty due to spatial rainfall variability, Geophys. Res. Lett., 34, L07403, doi:10.1029/2007GRL029409, 2007.

David, N., Alpert, P., and Messer, H.: Novel method for water vapor monitoring using wireless communication networks measurements, Atmos. Chem. Phys., 8, 11673-11684, 2008.

Feingold, G. and Levin, Z.: The lognormal fit to raindrop spectra from frontal convective clouds in Israel, J. Appl. Meteorol., 25, 1346-1363, 1986.

Goldshtein, O., Messer, H., and Zinevich, A.: Rain rate estimation using measurements from commercial telecommunications links, IEEE T. Signal Proces., 57(4), 1616-1625, 2009.

Grum, M., Kraemer, S., Verworn, H., and Redder, A.: Combined use of point rain gauges, radar, microwave link and level measurements in urban hydrological modeling, Atmos. Res., 77(14), 313-321, 2005.

Holt, A. R., Kuznetsov, G., and Rahimi, A. R.: Comparison of the use of dual-frequency and single-frequency attenuation for the measurement of path-averaged rainfall along a microwave links, IEE P-Microw. Anten. P., 150(5), 315-320, 2003.

Jameson, A.: A comparison of microwave techniques for measuring rainfall, J. Appl. Meteorol., (30), 32-54, 1991.

Kharadly, M. and Ross, R.: Effect of wet antenna attenuation on propagation data statistics, IEEE T. Antenn. Propag., 49(8), 1183-1191, 2001

Krystek, M. and Anton, M.: A weighted total least-squares algorithm for fitting a straight line, Meas. Sci. Technol., 18, 3438 3442, 2007.

Leijnse, H., Uijlenhoet, R., and Stricker, J.: Rainfall measurement using radio links from cellular communication networks, Water Resour. Res., 43(3), W03201, doi:10.1029/2006WR005631, 2007a.

Leijnse, H., Uijlenhoet, R., and Stricker, J.: Hydrometeorological application of a microwave link: 2. Precipitation, Water Resour. Res., 43, W04417, doi:10.1029/2006WR004989, 2007b.

Leijnse, H., Uijlenhoet, R., and Stricker, J.: Microwave link rainfall estimation: Effects of link length and frequency, temporal sampling, power resolution, and wet antenna attenuation, Adv. Water Resour., 31, 1481-1493, doi:10.1016/j.advwatres.2008.03.004, 2008a.

Leijnse, H., Uijlenhoet, R., and Stricker, J.: Sources of error in microwave link rainfall estimation, Proceedings of the International Symposium on Weather Radar and Hydrology, Grenoble, France, 2008b.

Messer, H., Zinevich, A., and Alpert, P.: Environmental monitoring by microwave communication networks, Science, 312, p. 713, 2006.

Minda, H. and Nakamura, K.: High temporal resolution pathaverage rain gauge with $50-\mathrm{GHz}$ band microwave, J. Atmos. Ocean. Tech., 22, 165-179, 2005.

Mishchenko, M.: Calculation of the amplitude matrix for a nonspherical particle in a fixed orientation, Appl. Optics, 39(6), 1026-1031, 2000

Papoulis, A.: Probability, random variables and stochastic processes, 3rd edn., McGraw-Hill, Singapore, 1991.

Press, W., Teukolsky, S., Vetterling, W., and Flannery, B.: Nu- 
merical recipes in C: the art of scientific computing, 2nd edn., Cambridge University Press, Cambridge, UK, 1992.

Rahimi, A. R., Holt, A. R., Upton, G. J. G., and Cummings, R. J.: The use of dual-frequency microwave links for measuring pathaveraged rainfall, J. Geophys. Res., 108(D15), ACL, 8-1, 2003.

Rincon, R. and Lang, R.: Microwave link dual-wavelength measurements of path average attenuation for the estimation of drop size distributions and rainfall, IEEE T. Geosci. Remote, 40(4), 760-770, 2002.

Schabenberger, O. and Gotway, C.: Statistical methods for spatial data analyses, Chapman \& Hall/CRC, Boca Raton, FL, USA, 2005.

Schleiss, M. and Berne, A.: Identification of dry and rainy periods using telecommunication microwave links, Proc. 34th AMS Conf. Radar Meteorol., Williamsburg, VA, USA, 2009.

Uijlenhoet, R., Porrà, J., Torres, D., and Creutin, J.-D.: Analytical solutions to sampling effects in drop size distribution measurements during stationary rainfall: Estimation of bulk rainfall variables, J. Hydrol., 328, 65-82, doi:10.1016/j.jhydrol.2005.11.043, 2006.
Upton, G. J. G., Cummings, R. J., Rahimi, A. R., and Goddard, J. W. F.: Microwave links: the future of urban rainfall measurement? Atmos. Res., 77(1-4), 300-312, 2005.

Widrow, B. and Kollár, I.: Quantization noise: roundoff error in digital computation, signal processing, control, and communications, Cambridge University Press, Cambridge, UK, 2008.

$\mathrm{Xu}, \mathrm{K}$. and Wilke, C.: A kernel-based spatio-temporal dynamical model for nowcasting weather radar reflectivities, J. Am. Stat. Assoc., 100(472), 1133-1144, 2005.

Zhang, W. and Moayeri, N.: Power-law parameters of rain specific attenuation, IEEE 802.16cc-99/24, 1999.

Zinevich, A., Messer, H., and Alpert, P.: Frontal rainfall observation by a commercial microwave communication network, J. Appl. Meteorol. Clim., 48(7), 1317-1344, doi:10.1175/2008jamc2014.1, 2009. 